\title{
Tuning Azoheteroarene Photoswitch Performance through Heteroaryl Design
}

\author{
Joaquín Calbo, ${ }^{\#, \dagger}$ Claire E. Weston, ${ }^{\#,+}$ Andrew J. P. White, ${ }^{\ddagger}$ Henry S. Rzepa, ${ }^{\ddagger}$ Julia Contreras-García, ${ }^{*}, \S, \|$ \\ and Matthew J. Fuchter*, $* 0$ \\ ${ }^{\dagger}$ Instituto de Ciencia Molecular, Universidad de Valencia, 46890 Paterna, Spain \\ ${ }^{\ddagger}$ Department of Chemistry, Imperial College London, London SW7 2AZ, United Kingdom \\ ${ }^{\S}$ Sorbonne Universités, UPMC Univ Paris 06, CNRS, Laboratoire de Chimie Théorique, CC 137 - 4, place Jussieu, F-75252 Paris \\ Cedex 05, France \\ "CNRS, UMR 7616, LCT, F-75005 Paris, France
}

\section{Supporting Information}

\begin{abstract}
Photoswitchable compounds, which can be reversibly switched between two isomers by light, continue to attract significant attention for a wide array of applications. Azoheteroarenes represent a relatively new but understudied type of photoswitch, where one of the aryl rings from the conventional azobenzene class has been replaced with a fivemembered heteroaromatic ring. Initial studies have suggested the azoheteroarenes-the arylazopyrazoles in particular-to have excellent photoswitching properties (quantitative switching and long $Z$ isomer half-life). Here we present a systematic computational and experimental study to elucidate the origin of the long thermal half-lives and excellent addressability of the arylazopyrazoles, and apply this understanding to determine important structure-property relationships for a wide array of comparable azoheteroaryl photoswitches. We identify compounds with $Z$ isomer half-lives ranging from seconds to hours, to days and to years, and variable absorption characteristics, all through tuning of the heteraromatic ring. Conformation perhaps plays the largest role in determining such properties: the compounds with the longest isomerization half-lives adopt a $\mathrm{T}$-shaped ground state $Z$ isomer conformation and proceed through a $\mathrm{T}$-shaped isomerization pathway, whereas the most complete photoswitching is achieved for compounds that have a twisted (rather than T-shaped) $Z$ isomer conformation. By balancing these factors, we report a new azopyrazole $3 \mathbf{p z H}$, which can be quantitatively switched to its $Z$ isomer (>98\%) with $355 \mathrm{~nm}$ irradiation, near-quantitatively (97\%) switched back to the $E$ isomer with $532 \mathrm{~nm}$ irradiation, and has a very long half-life for thermal isomerization $\left(t_{1 / 2}=74 \mathrm{~d}\right.$ at $\left.25{ }^{\circ} \mathrm{C}\right)$. Given the large tunability of their properties, the predictive nature of their performance, and the other functional opportunities afforded by usage of a heteroaromatic system, we believe the azoheteroaryl photoswitches to have huge potential in a wide range of optically addressable applications.
\end{abstract}

\section{INTRODUCTION}

Photoswitchable compounds, which can be reversibly switched between two isomers by light, continue to attract significant attention for a wide array of applications: from molecular motors, memory, and manipulators to solar thermal storage. ${ }^{1}$ While there are several molecular properties that impact upon photoswitch performance, two are of central importance: (1) the completeness of photoswitching at a given wavelength of light, and (2) the thermal stability of each switched state. Among the classes of photochromic compounds studied, the azobenzenes maintain a privileged position due to the ease of their synthesis, their excellent photoswitching properties (such as high extinction coefficient and quantum yields), and the fact that their isomerization results in a significant change in molecular shape. ${ }^{2}$

In general, a significant amount is known on how the chemical structure of photochromic compounds impacts their photochemical and thermal behavior. ${ }^{1 e, 2,3}$ In the case of azobenzenes, excellent progress has been made in tuning the properties of azobenzenes via the substituents on the aromatic ring(s). For example, the past few years have seen the successful design of azobenzene photoswitches that can be addressed with visible light, ${ }^{4}$ a property of particular importance for the rapidly growing field of photopharmacology. ${ }^{5}$ Tetra-ortho-substituted azobenzenes have shown high promise in this regard, such as the $o$-methoxy analogues reported by Woolley ${ }^{4 a, 6}$ and the $o$-fluoro compounds reported by Hecht. ${ }^{4 b, 7}$ Both can be switched with visible light due to separation of the $n-\pi^{*}$ transitions of the $E$ and $Z$ isomers, which allows the $E$ isomer to be switched at the long wavelength $n-\pi^{*}$ absorbance, rather than via the more usual $\pi-\pi^{*}$ transition (which generally requires UVA irradiation). In the case of the $o$-methoxyazobenzenes, separation of $n-\pi^{*}$ transitions is caused by a red-shift in the $E$ isomer transition due to unfavorable electronic interactions between the lone

Received: November 11, 2016

Published: December 23, 2016 
New photoswitches

3-pyrazoles<smiles>Cn1ccc(/N=N/c2ccccc2)n1</smiles><smiles></smiles>

5-pyrazoles<smiles>On1nccc1/N=N/c1ccccc1</smiles><smiles>C[18O]n1ncc(O)c1/N=N/c1ccccc1</smiles>

3-pyrroles<smiles>Cc1cc(N=Nc2ccccc2)c(O)n1C</smiles><smiles></smiles>

triazole and tetrazole ,<smiles>Cn1nnc(/N=N/c2ccccc2)n1</smiles>

Literature photoswitches

Fuchter et al ${ }^{11}$<smiles>Cn1cc(/N=N/c2ccccc2)cn1</smiles>

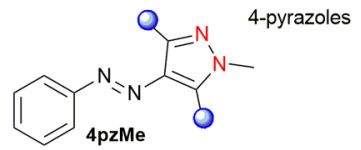<smiles>On1cccc1/N=N/c1ccccc1</smiles>

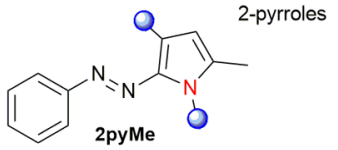
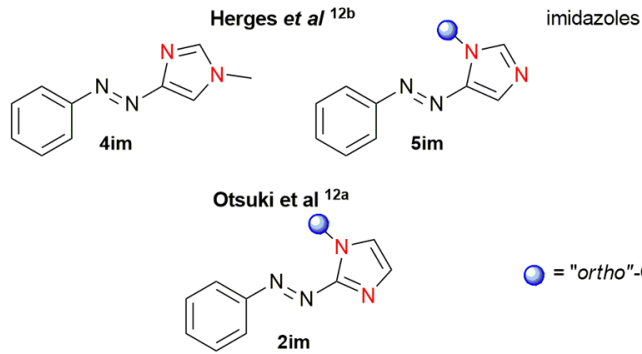

= "ortho"- $\mathrm{CH}_{3}$

Figure 1. Azoheteroaryl photoswitches considered in this study.

pairs of the methoxy and azo moieties, which raises the $n$ orbital energy. ${ }^{2 b}$ Meanwhile for the fluoroazobenzenes, the separation of the $n-\pi^{*}$ transitions is caused by a blue-shift in the $Z$ isomer transition due to a lowering of the $n$-orbital energy by the electron withdrawing fluorine substituents. ${ }^{4 \mathrm{~b}, 8}$

Increasing the thermal half-life of azobenzene $Z$ isomers, while retaining good addressability, ${ }^{9}$ has arguably been the most challenging endeavor for this class of photoswitch. Apart from the cyclic azoaryl switches, ${ }^{4 \mathrm{~d}, 10}$ the $Z$ isomers of azobenzenes are generally metastable and thermally revert back to the $E$ isomer over time. Once again, the tetra-orthosubstituted azobenzenes have provided the optimum solution to this problem, with the ortho-fluoroazobenzenes reported to have (in addition to excellent addressability) $Z$ isomer thermal half-lives of up to ca. 700 days $\left(25^{\circ} \mathrm{C}\right.$ in DMSO $){ }^{4 \mathrm{~b}, 7}$ This property was rationalized computationally as being partially caused by the increased stability of the $Z$ isomer, relative to the $E$ isomer (vs azobenzene), and partially due to a contribution of $Z$ isomer versus transition state (TS) dipole moments. ${ }^{4 \mathrm{~b}, 7}$

We have recently reported an alternative approach to access azo photoswitches with excellent properties (quantitative switching and long $Z$ isomer half-life) through replacement of one of the azobenzene aryl rings with a five-membered heteroaromatic ring. ${ }^{11}$ Such heteroaryl azo compounds are far less well studied ${ }^{12}$ than their ubiquitous azobenzene counterparts. Specifically, we found arylazopyrazoles $(4 \mathrm{pzH}$ and 4pzMe, Figure 1) to have excellent potential against the state of the art, as they could be quantitatively switched in one or both directions, depending on "ortho"-substitution, ${ }^{13}$ and had half-lives of 10 and 1000 days at $25^{\circ} \mathrm{C}$. Given this performance, the arylazopyrazoles are beginning to be employed in applications including in supramolecular complexes ${ }^{14}$ and as photopharmacological agents. ${ }^{15}$ The performance of the azoheteroaryl photoswitches is very dependent on the nature of the heteroaromatic ring, however, with the arylazopyrroles found to have shorter thermal half-lives $\left(21 \mathrm{~s}\right.$ and $12 \mathrm{~h}^{16}$ for 2pyMe and 2 pyH respectively, Figure 1) than the arylazopyrazoles; ${ }^{11}$ some other heteroaryl azo compounds have been found to have $Z$ isomer half-lives of microseconds to nanoseconds. ${ }^{12 \mathrm{c}}$ Clearly, a better understanding of how the photoswitching properties and performance can be tuned by a judicious choice of the type, positioning and substitution of the heteroaromatic ring would enable the azoheteroaryl photoswitches to reach their full potential. We note that such design "rules" should not only allow for optimization of intrinsic photoswitch performance, but also for the realization of designs where the heteroaromatic ring provides other functional opportunities. $^{17}$

There have been a large number of papers that use computational methods (see review by Burdette et al. ${ }^{2 a}$ ) to study the photochemical isomerization mechanisms of azobenzenes, although relatively few of these concern $Z-E$ thermal isomerization. ${ }^{18}$ Recently, Wegner and co-workers carried out experimental and computational studies into the thermal half-lives of sterically congested azobenzenes, and discovered that van der Waals forces play an important role. ${ }^{18 a}$ Favorable London dispersion interactions between ortho-alkyl substituents in the $Z$ isomers are responsible for an increase in $Z$ isomer stability upon increasing the bulkiness of the substituents. Comparatively, the azoheteroaryl photoswitches are almost unstudied in this regard. ${ }^{11,19}$ Only very recently, the $Z-E$ photoisomerization mechanism of two arylazopyrazoles has been computationally investigated. ${ }^{20}$ Notably, one of these compounds was found to exhibit near stereospecific excitedstate relaxation.

Herein we report a combined computational and experimental study on novel azoheteroaryl photoswitches derived from (nitrogen-based) five-membered heteroaromatic rings. We elucidate the origin of the long thermal half-lives and excellent addressability of the arylazopyrazoles, and apply this understanding to a wide array of comparable azoheteroaryl photoswitches. As such, we determine important structureproperty relationships as a function of the type of heteroaromatic ring, the connectivity of the ring (relative to the azo group), and the ring substitution. Furthermore, we identify that calculation of the azo $\mathrm{N}=\mathrm{N}$ Wiberg Index provides a quick and simple means to approximate the thermal half-live of this class of photoswitch, which can vary from 
seconds to days to months to years. This allows for rapid assessment of novel photoswitches based on ground state properties, importantly not relying on time-consuming transition state or excited state calculations. We believe these studies will greatly facilitate the further development of the azoheteroaryl photoswitches and their application to a broad array of functional areas.

\section{RESULTS AND DISCUSSION}

Thermal Half-Lives of the Z-Azoheteroaryl Photoswitches. Elucidation of the Factors Underpinning the $Z$ Isomer Thermal Half-Life for the Pyrrole and Pyrazole-Based Photoswitches. We commenced our studies using the previously described ${ }^{11}$ pyrrole and pyrazole photoswitches 2pyH, 2pyMe, 4pzH and 4pzMe (see Figure 1, and Table 1 for half-lives) as a small set of representative nitrogen-based azoheteroaryl photoswitches. Computational investigations were carried out in order to rationalize the structure-property relationships that had been observed experimentally, and to enable the discovery of other azoheteroaryl compounds with excellent photoswitching performance. Several conformations are available for such compounds, through rotation of the aromatic rings relative to the azo bridge. All the possible conformers of the $E$ and $Z$ isomeric forms were fully optimized using the hybrid exchange-correlation PBE0 functional ${ }^{21}$ including the Grimme's dispersion correction in its latest version (D3) ${ }^{22}$ and using the split-valence Pople's $6-31 G^{* *}$ basis set. ${ }^{23}$

Table 1. Theoretical Thermodynamic and Kinetic Parameters Including the Free Energy for the $Z-E$ Isomerization Reaction $\left(\Delta G_{Z-E}, \mathrm{kcal} / \mathrm{mol}\right)$ and the Free Energy Barrier $\left(\Delta G^{\ddagger}, \mathrm{kcal} / \mathrm{mol}\right)$ for the Lowest Energy Conformer of Each $Z$ Isomer, and the Weighted Average Half-Life Times (WA $t_{1 / 2}$ ) for All Four Possible TSs Compared with the Reported Experimental Values

\begin{tabular}{|c|c|c|c|c|}
\hline & $\Delta G_{Z-E}$ & $\Delta G^{\ddagger a}$ & WA $t_{1 / 2}$ & experimental $t_{1 / 2}$ \\
\hline $2 \mathrm{pyH}$ & -11.7 & $22.2(\mathrm{II})$ & $1.2 \mathrm{~h}$ & $12 \mathrm{~h}^{c}$ \\
\hline 2pyMe & -14.8 & 19.8 (II) & $120 \mathrm{~s}$ & $21 s^{b}$ \\
\hline $4 \mathrm{pzH}$ & -11.5 & $26.4(\mathrm{I})$ & 108 days & 1000 days $^{b}$ \\
\hline $4 \mathrm{pzMe}$ & -15.8 & 23.7 (I) & 1 day & 10 days $^{b}$ \\
\hline
\end{tabular}

${ }^{a}$ The type of the minimum-energy TS is indicated within parentheses. ${ }^{b}$ Data taken from Fuchter et al. ${ }^{11}{ }^{c}$ Lit. value of $3 \mathrm{~h}$ reported by Fuchter et al. ${ }^{11}$ was measured at $\sim 10^{-2} \mathrm{M}$ (see further discussion below).

Consistent with our previous study (Figure 1), ${ }^{11}$ compounds such as $\mathbf{2} \mathbf{p y H}$ and $4 \mathbf{p z H}$, which contain heteroaromatic rings that are not substituted in both the ortho positions, exhibit a $Z$ isomer conformation with a perpendicular $\mathrm{T}$ shape (Figure 2a). Such a T-shaped conformation is only accessible for azo compounds with five-membered heteroaromatic rings, and not for conventional azobenzenes. ${ }^{2 a, 12 b}$ The introduction of bulky groups in both ortho-positions of the heteroaromatic ring (such as in 2pyMe and 4pzMe) disfavors the T-shaped conformation and instead a twisted conformation is predicted, which has been crystallographically characterized for 4 pzMe. ${ }^{11}$ Compounds exhibiting twisted $Z$ isomer conformations are observed experimentally to have shorter $Z$ isomer half-lives relative to their less-hindered T-shaped analogues (Table 1). ${ }^{11}$ By introducing only one methyl group into the ortho position, such as is the case for $2 \mathbf{p y H}$, the two possible conformers can coexist (Figure 2a), and so both T-shaped and twisted a)

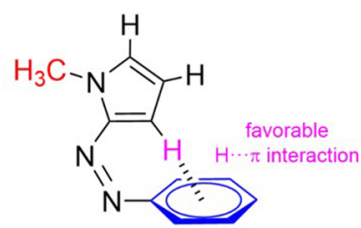

T-shape

b)

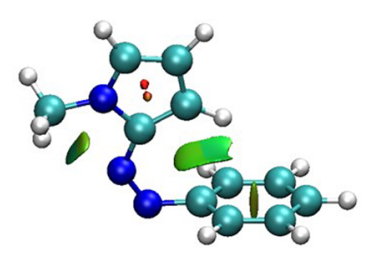

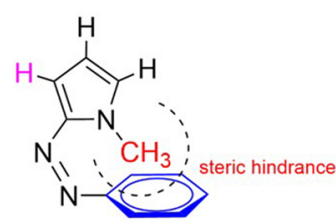

Twisted

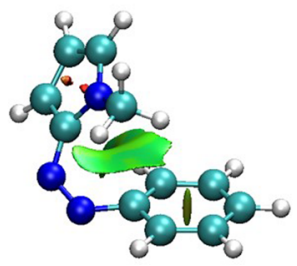

Figure 2. (a) The two possible $Z$ conformations when there is both a methyl group (bonded in this case to a nitrogen atom) and a hydrogen atom in the ortho positions, such as in $2 \mathrm{pyH}$. (b) The corresponding NCI surfaces: green areas indicate weak noncovalent interactions whereas narrow red regions designate steric ring clashes.

conformers need to be taken into account to provide accurate theoretical half-life times (vide infra).

From analysis of the relative enthalpies $\left(\Delta H_{Z-E}\right)$ between the $E$ and $Z$ minimum-energy conformations, the $Z$ isomer was found to be more than $10 \mathrm{kcal} / \mathrm{mol}$ less stable than the corresponding $E$ isomer for all the compounds examined (see Table S1 in the Supporting Information - SI). This difference increases upon introduction of two ortho methyls onto the heteroarene to ca. $13 \mathrm{kcal} / \mathrm{mol}$, which provides a thermodynamic driving force for accelerated isomerization. Given this, it was postulated that the increase in $Z$ isomer energy for 2 pyMe and 4pzMe was due to a destabilization of the $Z$ isomer ground state by the (sterically induced) twisted conformation. However, a closer inspection of the computed conformers reveals a subtle interplay of interactions, as can be visualized using a noncovalent index (NCI) analysis. ${ }^{24} Z$ isomer stabilization of derivatives containing at least one orthohydrogen on the heteroarene $(2 \mathrm{pyH}$ and $4 \mathrm{pzH})$ can occur through interactions present in the $\mathrm{T}$-shaped conformation, which are absent in the bis-o-methylated compounds (2pyMe and $4 \mathrm{pzMe})$. A localized surface arises between the orthohydrogen and the benzene ring (Figure $2 \mathrm{~b}$ ), which indicates the presence of a favorable $\mathrm{C}-\mathrm{H} \cdots \pi$ interaction. As a reference, the interaction energy for a T-shaped benzene dimer at the PBE0D3 $/ 6-31 \mathrm{G}^{* *}$ level is calculated to be $2.90 \mathrm{kcal} / \mathrm{mol}$. However, the twisted conformations of the bis-o-methylated compounds (2pyMe and $4 \mathrm{pzMe}$ ) exhibit a greater attractive NCI surface as a result of a larger dispersive contribution, despite the increased steric repulsion experienced (Figure 2b). When computed through Grimme's D3 approach, ${ }^{22}$ the dispersive contribution to the stability of the T-shaped $Z$ isomer compounds $2 \mathrm{pyH}$ and $4 \mathrm{pzH}$ is ca. $8 \mathrm{kcal} / \mathrm{mol}$, whereas it is ca. $11 \mathrm{kcal} / \mathrm{mol}$ in the case of the twisted methylated compounds 2pyMe and 4pzMe (see Table S1). For highly substituted azobenzenes, ${ }^{18 a}$ the dispersive contribution has been reported to overcome the steric repulsion and stabilize the $Z$ isomer, increasing the isomerization half-life. Similarly, in the azoheteroaryl compounds, the computational investigation of heteroarene substituents more bulky than a methyl group was found to result in stabilizing dispersive interactions in the $Z$ isomer that surpass any steric congestion (see Table S2 and Figure S24 in the SI). Thus, there 
appears to be a compensation principle in effect where the introduction of substituents destabilizes the structure through steric clashes, but the substituted ring adopts a conformation that maximizes mutual stabilizing dispersive interactions. Despite this, the destabilizing steric effect appears to dominate for the systems under primary study here (ortho $\mathrm{H}$ vs $\mathrm{Me}$ ), with the (twisted) substituted heteroarene $Z$ isomers (2pyMe and 4pzMe) being higher in energy than comparable compounds with $\mathrm{T}$-shaped $Z$ isomer conformers (2pyH and $4 \mathrm{pzH}$ ).

The computed conformation-induced differences in the relative energies between $E$ and $Z$ isomers do not however correlate with the half-live trends observed experimentally (Table 1). For example, calculations of the free energy for $Z-$ $E$ isomerization indicate that the $Z$ isomer of 4 pzMe is 15.8 $\mathrm{kcal} / \mathrm{mol}$ less stable than the $E$ isomer, whereas in $2 \mathrm{pyH}$ the difference is slightly smaller $(11.7 \mathrm{kcal} / \mathrm{mol})$; however, the halflives of the $Z$ isomers of these compounds follow the opposite trend: 10 days and $12 \mathrm{~h}$, respectively. It would therefore appear that there is a significant kinetic component to the thermal isomerization rates observed.

Given this, transition states (TS) and activation barriers $\left(\Delta G^{\ddagger}\right)$ were computed for the different $Z-E$ isomerization processes at the DFT PBE0-D3/6-31G** level of theory. Two principle mechanistic pathways for azobenzene isomerization have been previously reported-rotation about the $\mathrm{N}=\mathrm{N}$ bridge and inversion at one of the azo nitrogen atomshowever, variations on these have also been suggested. ${ }^{2 a}$ In the case of azoheteroaryl photoswitches, the inversion mechanism was found to be the lowest energy pathway for the thermal isomerization of all the compounds analyzed (see Figures S25 and S26 for further details). Inversion could occur either at the azo nitrogen bearing the phenyl ring (type-I, Figure 3a) or at the azo nitrogen bearing the heteroaryl ring (type-II). Moreover, and in accordance with the conformational possibilities afforded by these novel photoswitches, the type-I TS is reminiscent of the $Z$ isomer ground state conformation, where the heteroaryl ring is (almost) perpendicular to the benzene ring, whereas the type-II TS is closer to the $E$ isomer conformation, where the two aromatic rings are (almost) coplanar. Since there are two possible conformations for the inversion transition state when the system is nonsymmetric on either side of the azo group (Figure 3a), there are four possible transition states to be considered. Analysis of all these TSs yielded, in general, similar energetic barriers for the four possible pathways. Changing the computational method, the basis set, or moving from the gas phase to a continuum solvent model for acetonitrile all had negligible effect on the computed relative energy barriers (Table S3). All the pathways were therefore weight-averaged to obtain mean half-lives according to a Boltzmann distribution of the TS energy barriers, assuming an equally balanced ratio of the two $Z$ conformers. ${ }^{25}$

Theoretical evaluation of the free energy was carried out in gas phase, including thermal corrections to the total partition function at $298.15 \mathrm{~K}$. Using transition state theory, ${ }^{26}$ the rate constants for isomerization were calculated, assuming a firstorder reaction, and from this we computed the final weightedaverage half-lives (WA $t_{1 / 2}$ in Table 1 ). Despite the reasonably small energy differences (in the range of few $\mathrm{kcal} / \mathrm{mol}$ ) calculated for the transition state barriers of the different compounds, large differences in the half-lives for the photoswitches are predicted, which show good agreement with the available experimental data (Table 1). a)

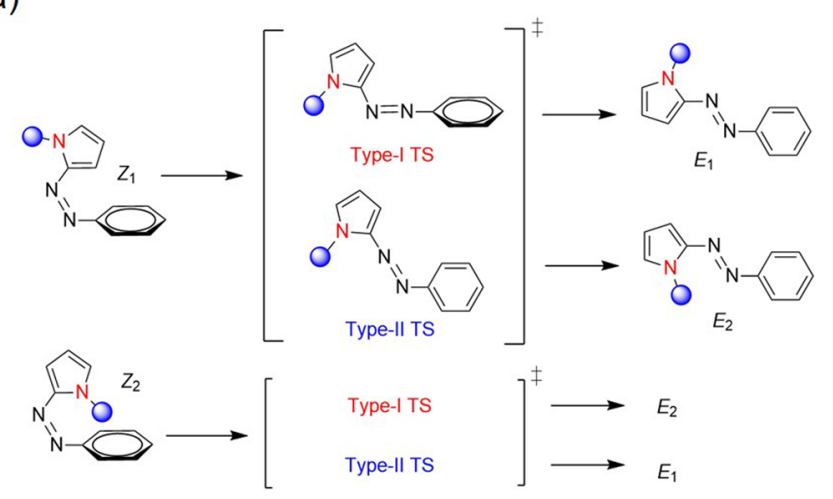

b)

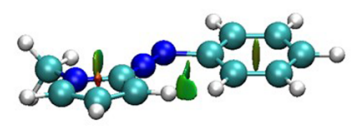

2pyH (II)

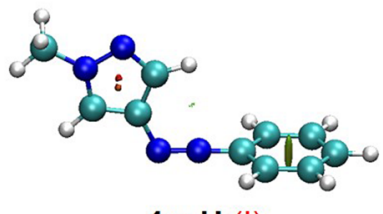

$4 \mathrm{pzH}(\mathrm{I})$

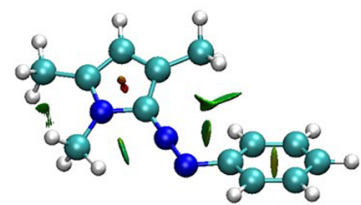

2pyMe (II)

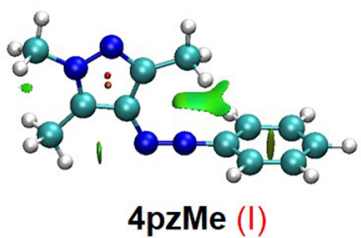

Figure 3. (a) The two lowest energy $Z-E$ thermal isomerization pathways of the representative photoswitch $2 \mathrm{pyH}$. (b) NCI surfaces calculated from the PBE0-D3/6-31G** electron density for the TS of compounds 2pyH, 2pyMe, $4 \mathrm{pzH}$ and $4 \mathrm{pzMe}$ that lead to the lowest free energy barriers. The TS type (either I or II) is indicated within parentheses.

NCI analysis was employed to disentangle the effects underpinning this energetic ordering. Pyrroles 2pyH and 2pyMe exhibit the lowest TS energy barriers and a TS similar to the $E$ isomer conformation, where the two aromatic rings attempt to adopt a coplanar relationship (type-II). The presence of the ortho methyl group in 2pyMe results in more significant twisting away from such coplanarity relative to 2pyH. The NCI surfaces of the pyrrole TS (Figure 3b) show a number of stabilizing dispersive interactions. For example, when quantified through Grimme's approach, we find the stabilizing dispersive contribution to compound 2pyMe to be $10.2 \mathrm{kcal} / \mathrm{mol}$, compared with a smaller value of $7.1 \mathrm{kcal} / \mathrm{mol}$ for 2pyH. The lowest-energy TS for pyrazoles $4 \mathrm{pzH}$ and 4pzMe has a T-shaped conformation, similar to the $Z$ isomer ground state conformation, where the heteroaryl ring is perpendicular to the phenyl ring (type-I). Interestingly, NCI analysis indicates dispersion is almost absent in the (lowestenergy) TS of $\mathbf{4 p z H}$ (Figure $3 \mathrm{~b}$ ); the dispersive contribution is calculated to be only $6.2 \mathrm{kcal} / \mathrm{mol}$ (see Table S1). As in the case of the ground state conformations, methyl substitution at the ortho position leads to greater dispersive effects. These results suggest that the TSs are stabilized by noncovalent interactions, mainly dispersion, and such interactions determine the TS energetics: compound 2pyMe has the highest stabilization through dispersion and therefore the lowest energy 
Table 2. Experimental $Z-E$ Thermal Isomerisation Kinetics at $25{ }^{\circ} \mathrm{C}$, Including the Remeasured Values for Literature Compounds $2 \mathrm{pyH}$, $4 \mathrm{im}$ and $5 \mathrm{im}$

\begin{tabular}{|c|c|c|c|c|}
\hline & $\begin{array}{l}\text { Position of azo } \\
\text { on heteroarene }\end{array}$ & & Rate $(\mathrm{k}) / \mathrm{s}^{-1}$ & Half-life $\left(t_{1 / 2}\right)$ \\
\hline tetrazole & 4 & tet $^{a}$ & $2.1 \times 10^{-7}$ & $39 d$ \\
\hline triazole & 3 & 3 tri $^{a}$ & $3.8 \times 10^{-7}$ & $21 \mathrm{~d}$ \\
\hline \multirow{6}{*}{ pyrazoles } & 3 & $3 p z H^{a}$ & $1.1 \times 10^{-7}$ & $74 \mathrm{~d}$ \\
\hline & 3 & $3 p z M e^{b}$ & $1.5 \times 10^{-6}$ & $5.8 \mathrm{~d}$ \\
\hline & 4 & $4 p_{z} H^{a, e}$ & $7.6 \times 10^{-9}$ & $1000 \mathrm{~d}$ \\
\hline & 4 & $4 \mathrm{pzMe} \mathrm{M}^{\mathrm{b}, \mathrm{e}}$ & $7.7 \times 10^{-7}$ & $10 d$ \\
\hline & 5 & $5 \mathrm{pzH}^{\mathrm{b}}$ & $7.4 \times 10^{-7}$ & $11 \mathrm{~d}$ \\
\hline & 5 & $5 p z M e^{c}$ & $1.6 \times 10^{-5}$ & $12 \mathrm{~h}$ \\
\hline \multirow{4}{*}{ pyrroles } & 2 & $2 \mathrm{pyH}^{\mathrm{c}, \mathrm{f}}$ & $6.5 \times 10^{-5}$ & $12 \mathrm{~h}$ \\
\hline & 2 & $2 p y M e^{c, d, e}$ & $3.3 \times 10^{-2}$ & $21 \mathrm{~s}$ \\
\hline & 3 & $3 p H^{c, d}$ & $2.2 \times 10^{-6}$ & $3.7 d$ \\
\hline & 3 & 3pyMe ${ }^{c, d}$ & $7.6 \times 10^{-4}$ & $0.25 \mathrm{~h}$ \\
\hline \multirow{3}{*}{ imidazoles } & 2 & $2 \mathrm{im}^{\mathrm{g}}$ & $2.2 \times 10^{-5}$ & $9 \mathrm{~h}$ \\
\hline & 4 & $4 \mathrm{im}^{\mathrm{chh}}$ & $3.3 \times 10^{-6}$ & $2.4 \mathrm{~d}$ \\
\hline & 5 & $\operatorname{sim}^{c, i}$ & $1.2 \times 10^{-6}$ & $6.5 \mathrm{~d}$ \\
\hline
\end{tabular}

Measured using: ${ }^{a} \mathrm{NMR}$ at a range of elevated temperatures, $\sim 10^{-2} \mathrm{M}$ in DMSO- $d_{6} \cdot{ }^{b} \mathrm{NMR}, \sim 10^{-2} \mathrm{M}$ in $\mathrm{MeCN}-d_{3} \cdot{ }^{c} \mathrm{UV}-\mathrm{vis}, 10^{-5} \mathrm{M}$ in anhydrous

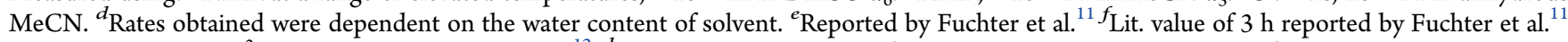
was measured at $\sim 10^{-2} \mathrm{M} .{ }^{g}$ Reported by Otsuki et al. ${ }^{12 \mathrm{a}}{ }^{h} \mathrm{~A}$ thermal half-life of $5 \mathrm{~h}$ (solvent or concentration not known) was reported by Herges et al. ${ }^{12 \mathrm{~b}} \mathrm{~A}$ thermal half-life of $22 \mathrm{~d}$ (solvent or concentration not known) was reported by Herges et al. ${ }^{12 \mathrm{~b}}$

barrier, whereas compound $4 \mathrm{pzH}$ has the lowest dispersion stabilization and therefore the highest TS energy.

In summary, the isomerization rate (and therefore the halflives) of photoswitches $2 \mathrm{pyH}, 2 \mathrm{pyMe}, 4 \mathrm{pzH}$ and $4 \mathrm{pzMe}$ are controlled by both the $Z$ isomer ground state stabilization and the TS energy for a given isomerization process. In general, the pyrazoles appear to have much longer isomerization half-lives than the pyrroles due to their preference to adopt T-shaped (type-I) TSs, which lack significant dispersive stabilization and hence have a higher TS energy. Methyl substitution in the ortho position twists the ground state $Z$ isomer conformations of both the pyrroles and pyrazoles away from the T-shape which, through steric effects, destabilizes the ground state $Z$ isomer of 2 pyMe and $4 \mathrm{pzMe}$ relative to $2 \mathrm{pyH}$ and $4 \mathrm{pzH}$, respectively. The compound with the longest thermal $Z$ isomer half-life, 4pzH (1000 days), exhibits both a T-shaped ground state $Z$ isomer conformation and proceeds through a T-shaped isomerization pathway. The role of the $\mathrm{T}$-shape conformation in these processes is therefore crucial.

Synthesis and Characterization of New Azoheteroaryl Photoswitches. In order to elucidate structure-property relationships for a wider array of azoheteroaryl photoswitch systems and provide predictive trends to enable future azoheteroaryl photoswitch design, it was necessary to synthesize and characterize a larger number of analogues (Figure 1). These were considered in comparison with $\mathbf{2} \mathbf{p y H}$, 2pyMe, 4pzH and 4pzMe, and with the phenylazoimidazole photoswitches previously characterized in the literature. ${ }^{12 a, b}$ The principle variables considered when selecting novel candidate compounds for synthesis were: (i) the number and positioning of the nitrogen atoms (and therefore formally the heteroaryl ring present); and (ii) the number and positioning of heteroaryl methyl substituents. The new analogues (Figure 1) were synthesized using similar routes to those previously described (see SI). ${ }^{11}$

Thermal isomerization rates and half-lives were measured for the $Z$ isomers of the newly synthesized compounds (Table 2, which includes the literature compounds for comparison). Following $E-Z$ switching at $355 \mathrm{~nm}$ (vide infra), rates at 25 ${ }^{\circ} \mathrm{C}$ were obtained using either UV-vis or NMR spectroscopy (Figures S15-18): the kinetics of analogues with half-lives of less than 1 day (or those showing a concentration-dependence - vide infra) were measured using UV-vis spectroscopy at a concentration of $1 \times 10^{-5} \mathrm{M}$ in acetonitrile. Otherwise, kinetics 
were more conveniently measured by NMR spectroscopy at $\sim 10^{-2} \mathrm{M}$; either at $25{ }^{\circ} \mathrm{C}$ in acetonitrile- $d_{3}$ or, for analogues with half-lives of longer than 10 days at $25{ }^{\circ} \mathrm{C}$, (for example tet, 3 tri, $3 \mathrm{pzH})$, at a range of higher temperatures $\left(70-110^{\circ} \mathrm{C}\right)$ in DMSO- $d_{6}$. For the latter cases, $\Delta H^{\ddagger}$ and $\Delta S^{\ddagger}$ values were extracted using Eyring plots, and from these, the isomerization rates and half-lives at $25^{\circ} \mathrm{C}$ were calculated (see SI).

Clear experimental trends are apparent from the data summarized in Table 2. When comparing compounds with the same azo linkage position on a given heteroarene series, the bis-ortho-methylated photoswitches had faster thermal isomerization rates than their singly or nonmethylated analogues, consistent with the analysis above. The position of the ring nitrogen atom(s) also had a significant effect on the rate. By separating the photoswitches into those with $\leq 1$ ortho-methyl groups and those with two ortho-methyl groups, the 2azopyrroles had shorter half-lives than the corresponding 3azopyrroles $\left(t_{1 / 2}: 2\right.$ pyMe $<3$ pyMe, 2 pyH $<3$ pyH $)$, and for the azopyrazoles, the 5 -analogues had the shortest half-lives and the 4-analogues had the longest half-lives $\left(t_{1 / 2}: 5 \mathbf{p z H}<3 \mathbf{p z H}<\right.$ 4pzH, 5pzMe $<3$ pzMe $<4$ pzMe). It should be noted that although 3pzMe has been included in the pzMe series, it differs from the other compounds (5pzMe and 4pzMe) in that it has only one ortho-methyl and a basic nitrogen in the other orthoposition (as opposed to two ortho-methyls). The finding that this compound fits into the pzMe series suggests that ortho basic nitrogens have a similar effect on the thermal isomerization rate as $o$-methylation. Consistent with this, the presence of a nitrogen atom in the ortho position was computationally found to disfavor the T-shaped conformation, and results in a twisted conformation of the $Z$ isomer (Figure 4). For the

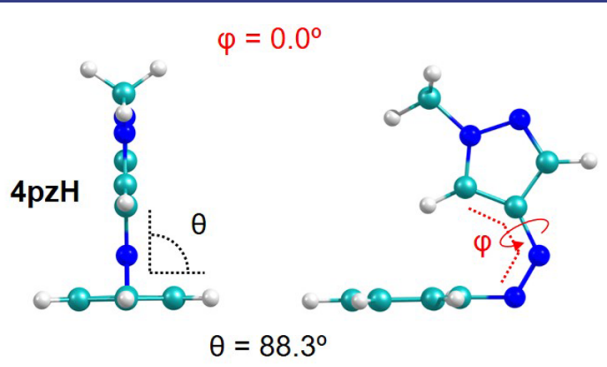

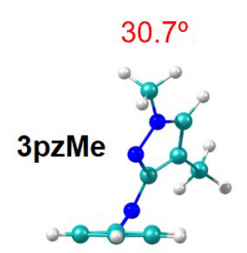

$60.6^{\circ}$

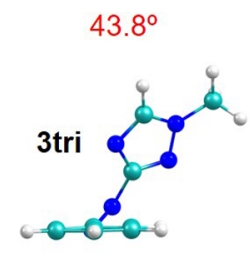

$52.1^{\circ}$ $49.2^{\circ}$

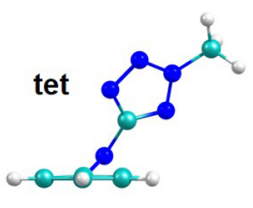

$49.9^{\circ}$
Figure 4. Characteristic dihedral angles of the lowest energy conformations of representative $Z$ isomer compounds with basic nitrogen atoms in the ortho position of the heteroarene (bottom) compared with the long-living T-shaped $Z$ isomer of photoswitch 4pzH (top).

imidazole compounds, $4 \mathrm{im}$ and $5 \mathrm{im}$ both have one orthohydrogen so can be considered as part of the same series $\left(t_{1 / 2}\right.$ : 5im $>$ 4im) due to the formation of a T-shaped conformation (as discussed by Herges and co-workers). ${ }^{12 \mathrm{~b}}$ 2im however has one ortho-methyl and one ortho basic nitrogen so adopts a twisted conformation.
More Extensive Structure Property Relationships: Computational vs Experimental Trends. An analogous computational treatment to that described for compounds 2pyH, 2pyMe, 4pzH and 4pzMe was performed on all the newly synthesized compounds, as well as the other previously reported azoheteroaryl photoswitches in the literature. Comparison of the $Z-E$ isomerization half-lives obtained experimentally to those computed (taking into account both thermodynamic and kinetic factors) is shown in Figure 5. In general, the calculated

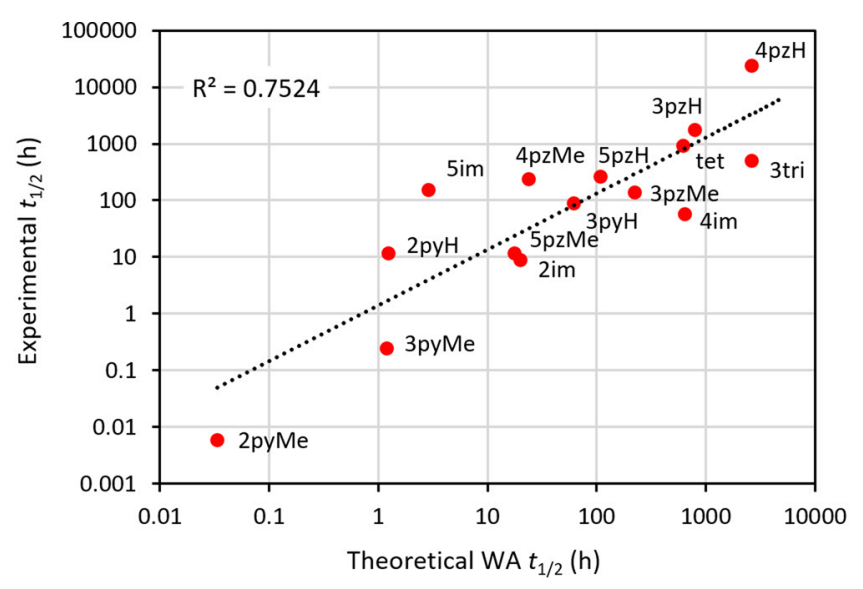

Figure 5. Comparison between the theoretical vs experimental half-life times for the list of photoswitches under study. This data uses the revised thermal lifetimes for 4im and $5 \mathrm{im}$ (see text).

half-lives were good predictions of the experimental values (i.e., within the same order of magnitude), and the majority of halflives were predicted in the correct order, shortest to longest (Figure 5). The comparison of the theoretical vs experimental half-lives for this larger array of compounds confirms the physical effects (outlined above) that underpin the thermal halflife of these azoheteroaryl photoswitches: compounds that adopt a more $\mathrm{T}$-shaped conformation, such as $\mathbf{3 p y H}$ and $\mathbf{5 p z H}$, had slower thermal isomerization rates than their twisted analogues (3pyMe and 5pzMe). In addition to this, the effect seen upon changing the position of the azo linkage on the heteroarene was correctly predicted for the pyrroles and pyrazoles, with the sole exception of 3pzMe. Experimentally, the order of increasing half-life for this series was 5pzMe < $3 p z M e<4 p z M e$, whereas the calculated half-life for 3pzMe was longer than 4pzMe. As discussed above, this compound differs from the other azopyrazoles studied as it has one orthomethyl and one ortho basic nitrogen, rather than two o-methyls or one o-methyl and one ortho $\mathrm{CH}$ group. The azo-positional effect can be rationalized by the extent of conjugation between the heteroarene and the phenylazo moiety (vide infra).

In our initial comparison of the experimental and computed half-lives, there were two compounds (4im and 5im)previously reported by Herges and co-workers ${ }^{12 b}$ - for which the predicted values were observed to be notably more divergent than the experimental values obtained from the literature. Since there was no apparent reason why these compounds in particular should be outliers, combined with the fact that the concentrations or solvents in which half-lives were measured were not reported, we decided to synthesize 4im and 5im and remeasure their thermal half-lives. The compounds were synthesized following the literature method reported by Herges. ${ }^{12 b}$ Confirmation of the structures of isomeric compounds $4 \mathrm{im}$ and $5 \mathrm{im}$, including unambiguous assignment 
'COMPLETE' HETERARENE CONJUGATION

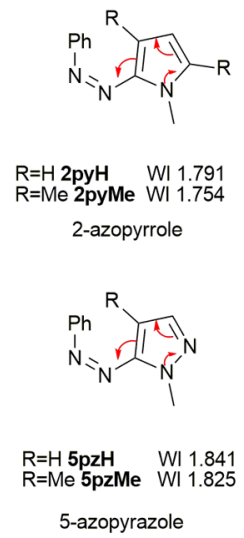

'PARTIAL' HETEROARENE CONJUGATION

$$
\begin{aligned}
& \mathrm{R}=\mathrm{H} \text { 3pyH } \quad \mathrm{Wl} 1.828 \\
& \mathrm{R}=\mathrm{Me} \text { 3pyMe Wl } 1.822 \\
& \text { 3-azopyrrole }
\end{aligned}
$$

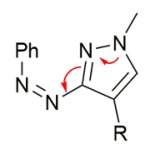

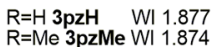

3-azopyrazole

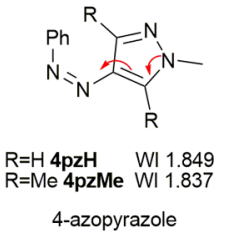

Figure 6. Conjugation of the heteroarene into the azophenyl moiety. WI = Wiberg index.

of $4 \mathrm{im}$ by X-ray crystallography (see Figure S2 in the SI), was consistent with the previous structural assignment. ${ }^{12 \mathrm{~b}} \mathrm{An}$ interesting effect was observed however when attempts were made to determine the thermal isomerization rates of $4 \mathrm{im}$ and 5im: the measured rate of $4 \mathrm{im}$ was concentration-dependent, with faster rates observed at high concentrations $\left(\gg 10^{-4} \mathrm{M}\right)$ (Table 2). Although the origin of this effect is unclear, it seems that there may be bimolecular pathways at higher concentration that facilitate isomerization for this compound. At concentrations below $10^{-3} \mathrm{M}$, no concentration dependence on the half-life was observed. 5im did not show a concentration dependence; however, under the conditions of this study $\left(10^{-5}\right.$ $\mathrm{M}$ concentration in acetonitrile, $25^{\circ} \mathrm{C}$ ) a shorter half-live was obtained than that previously reported. ${ }^{12 \mathrm{~b}}$ The half-lives for 4im and 5im, when measured at the same concentration of the majority of the other photoswitches included in this study $\left(10^{-5} \mathrm{M}\right.$, see Table 2$)$ were 2.4 and $6.5 \mathrm{~d}$, respectively (cf., the reported values of $5 \mathrm{~h}$ and $22 \mathrm{~d}$, which were presumably carried out under different conditions). The inclusion of these revised values into our comparative analysis gave an improved fit (Figure 5).

In light of the discovery of a concentration dependence on the thermal half-life, we examined whether other compounds under study exhibited a comparable effect. To complete the azoimidazole series, $2 \mathrm{im}$ was also synthesized and found to have the same half-life when measured at $10^{-5} \mathrm{M}$ (by UV-vis) and at $10^{-2} \mathrm{M}$ (by NMR), which matched the value reported by Otsuki and co-workers $\left(t_{1 / 2}\right.$ of $\left.9 \mathrm{~h}\right) .^{12 a}$ For the novel photoswitches synthesized in this study, a representative set ${ }^{27}$ was examined and it was found that the azopyrroles show a concentration dependence, whereas the azopyrazoles do not (see Figures 19-22 in the SI for more details). Specifically, the published half-life of $2 \mathrm{pyH}$ was $3 \mathrm{~h}$ when recorded at $\sim 10^{-2} \mathrm{M}$ and $12 \mathrm{~h}$ when measured at $10^{-5} \mathrm{M}$, whereas it shows negligible concentration dependence at concentrations below $\sim 10^{-3} \mathrm{M}$. This observation is crucial when designing new photoswitches and envisaging their technological use: concentration may be a useful parameter for tuning switch properties.

It was also found that the azopyrroles are sensitive to water content in the solvent, where increased water content leads to accelerated thermal isomerization (as reported previously for 2 pyMe ${ }^{28}$ ), whereas the other heteroaryl systems were not. Although the origin of this effect has not been further studied, it is plausible that polar isomerization transition states are more stabilized in the presence of water, thus leading to faster isomerization. The fact that pyrroles are good electron donors $\left(\sigma_{\mathrm{Ar}}^{+}=-1.96 \text { for } N \text {-methyl-2-pyrrolyl }\right)^{29}$ into the azophenyl moiety may lead to the formation of a more polar TS for heteroaryl photoswitches. Theoretical calculations confirmed that the pyrrole-based transition states have larger electrondonor character than the pyrazole analogues, and that this difference is more accentuated upon inclusion of water solvent effects (Figure S27).

Rapid Approximation of $Z$ Isomer Thermal Half-Life. While in-depth computational study of the thermodynamics and kinetics of the isomerization process provides insight into the structure-property effects of the azoheteroaryl photoswitches, and allows for assessment of the magnitude of the $Z$ isomer thermal half-life, such an analysis clearly requires TS structures to be obtained; which is not practical for large screening of diverse sets of prospective new heteroaromatic photoswitch designs. We therefore sought to define a predictive metric that would be simpler/quicker to calculate and inform on likely photoswitch performance. We selected calculation of the $\mathrm{N}=\mathrm{N}$ bond order (the Wiberg index ${ }^{30}$ or WI) of the optimized $Z$ conformers as a potentially useful metric. Bond orders were calculated as the sum of the off-diagonal square of the density matrix obtained at the PBE0-D3/6-31G** level (see the SI for more details), and averaged for the two possible $Z$ isomer conformations of each photoswitch. As expected, the WIs obtained show a linear correlation with respect to the azo $\mathrm{N}=\mathrm{N}$ bond length (Figure S28a). In all systems studied, the $\mathrm{N}$ (azo)-C(benzene) bond length is computed to be larger than the $\mathrm{N}(\mathrm{azo})-\mathrm{C}$ (heteroarene) bond length. The difference between these two magnitudes (NC asymmetry) ${ }^{18 \mathrm{f}}$ is also predicted to correlate with the $\mathrm{N}=\mathrm{N}$ bond length (Figure $\mathrm{S} 28 \mathrm{~b}$ ). Since the $\mathrm{N}=\mathrm{N}$ bond length highly depends on the extent of $\pi$-conjugation in the photoswitch, WI calculations will give valuable information on the strength of the $\mathrm{N}=\mathrm{N}$ double bond and, presumably, on the relative half-life times.

Compounds that exhibit a twisted $Z$ isomer conformation, due to ortho-substitution, were found to result in elongation of both the $\mathrm{N}-\mathrm{C}$ hindered bond, and the $\mathrm{N}=\mathrm{N}$ azo bond, with a concomitant decrease in the bond order (smaller Wiberg index). This was particularly noticeable for the bis-o-methylated 2pyMe and 4pzMe when compared to their less hindered analogues $\mathbf{2 p y H}$ and $\mathbf{4 p z H}$ (Figure 6). The nature and connectivity of the heteroaromatic ring was found to impact more noticeably on the Wiberg index calculated. For example, the 2-pyrroles have lower Wiberg indices (e.g., 2pyH, and 
2 pyMe) than the 3-pyrroles and all of the pyrazoles. The effect upon changing the connectivity of the heteroaryl ring (or conversely viewed as changing the azo positioning) can be qualitatively explained in terms of conjugative electron donation from the $N$-methyl lone pair to the azophenyl system (Figure 6). For the 2-azopyrroles and the 5-azopyrazoles, the position of the $\mathrm{N}$-methyl relative to the azo group allows more extensive conjugation of the lone pair into the azophenyl relative to the other regioisomers. This improved electron donation from the heteroaryl ring likely leads to a decrease in the bond order through polarization of the $\mathrm{N}=\mathrm{N}$ bond (pushpull type effect). Similar electronic effects are apparent upon changing the type of heteroaryl ring: for example, pyrroles $\left(\sigma_{\mathrm{Ar}}{ }^{+}\right.$ $=-1.96, \mathrm{~N}$-methyl-2-pyrrolyl $)^{29}$ are better electron donors into the azo group than pyrazoles $\left(\sigma_{\mathrm{Ar}}^{+}=-0.41,-0.99,-0.29\right.$ for $\mathrm{N}$-methyl-3-pyrazolyl, $\mathrm{N}$-methyl-4-pyrazolyl, and $\mathrm{N}$-methyl-5pyrazolyl respectively), ${ }^{31}$ and hence have lower Wiberg indices (bond orders) relative to the pyrazoles.

Comparison of the experimental $t_{1 / 2}$ values with the Wiberg indices for all the azoheteroaryl photoswitches studied is shown in Figure 7. Despite the simplified nature of the Wiberg index

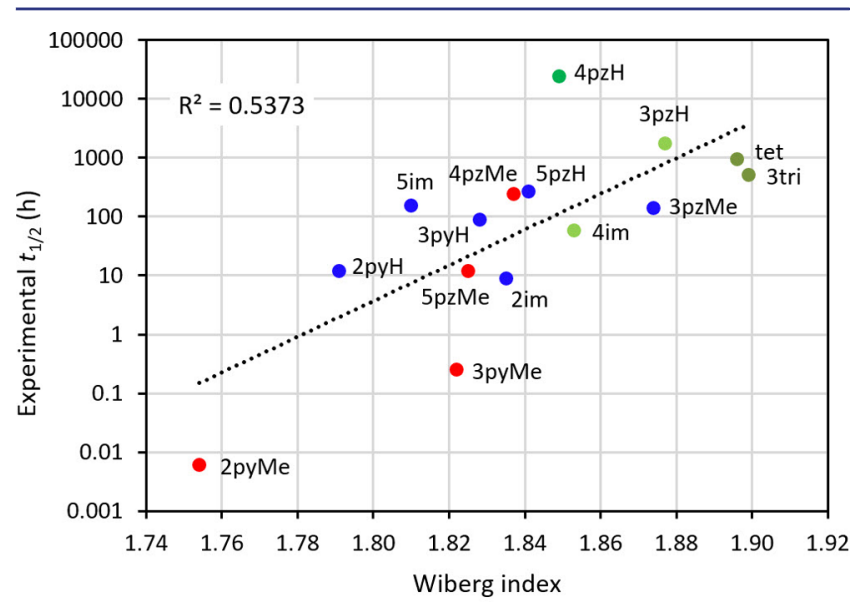

Figure 7. Correlation between the experimental half-lives and the Wiberg index colored by families: bis-ortho-methylation (red), monoortho-methylation (blue), and no ortho-methylation (green). The last family is subdivided in the number of basic nitrogen atoms in orthoposition (light, one atom; medium, zero atoms; and dark, two atoms).

to approximate the structural and conformational effects of this diverse range of compounds, a clear trend appears, although residuals are significant $\left(R^{2}=0.54\right)$. When compared with the theoretical half-life times, the correlation is more significant $\left(R^{2}\right.$ $=0.82$, see Table S4 and Figure S29). Photoswitches with "partial" heteroarene conjugation (Figure 6), such as $3 \mathrm{pzH}$, 3tri, tet, etc., are predicted to provide the largest Wiberg indices, correlating well with long experimental half-lives. On the other hand, "complete" heteroarene conjugated systems, such as $\mathbf{2 p y H}$, 2 pyMe, 5 pzMe, are calculated with the lowest Wiberg indices out of the compounds studied, which correlates well with their short half-life values. The largest outliers in this trend are those most impacted by ortho substitution or lack thereof. For example, photoswitch 4 pzH has a calculated halflife 2 orders of magnitude larger than expected from its Wiberg index. As discussed above, its lack of ortho substitution allows for a T-shaped $Z$ isomer ground state and T-shaped TS barrier. In contrast, photoswitch 3pyMe exhibits a half-life nearly 2 orders of magnitude shorter than what is expected from its
Wiberg index. In this case, the bis-ortho-substitution more significantly destabilizes the $Z$ isomer with respect to the transition state, permitting an unexpectedly fast $Z-E$ isomerization (see also compound 2pyMe). Other key outliers include those with concentration dependent half-lives, such as $2 \mathrm{pyH}$ and $5 \mathrm{im}$. We note that when $4 \mathrm{pzH}, 2 \mathrm{pyH}$, and $5 \mathrm{im}$ are removed from the comparison, the trend with respect to experimental $t_{1 / 2}$ values vs the Wiberg index is much improved $\left(R^{2}=0.76\right)$.

$Z-E$ Photoisomerization. The UV-vis absorbances of azoarene $E$ and $Z$ isomers determines their practical utility: their addressability. ${ }^{9}$ Overlapping absorbances in the characteristic electronic transitions between such isomers leads to incomplete photoswitching. Therefore, suitable separation of the $\lambda_{\max }$ values for transitions associated with each isomer is highly desirable for good/quantitative two-way photoswitching at defined wavelengths. The low-energy $n-\pi^{*}$ band is often exploited for $Z-E$ photoisomerisation of azobenzenes; however, there is frequently overlap of the absorbances between the two isomers in this spectral window. Several modified azobenzenes, such as Woolley's $o$-methoxy ${ }^{4 a, 6 a}$ and Hecht's $o$-fluoroazobenzenes, ${ }^{4 \mathrm{~b}, 7}$ have been reported in which the $n-\pi^{*}$ band of the two isomers are sufficiently well separated to allow photoswitching for both $E-Z$ and $Z-E$ isomerization using this transition. In particular, several of the $o$-fluoroazobenzene analogues developed by Hecht have improved (near quantitative) photoconversion ( $\leq 95 \% Z$ and $\leq 97 \% E$ ) to those usually seen for azobenzenes.

In our initial study, ${ }^{11}$ we showed that the nature (pyrrole versus pyrazole) and ring (methyl) substitution of four azoheteroaryl compounds significantly impacts their absorption spectra and, in turn, their photoswitching behavior. Most notably, we found that excitation of the $\pi-\pi^{*}$ transition of $E$ 4 pzMe at $355 \mathrm{~nm}$ resulted in complete photoswitching (>98\% $Z$ isomer), whereas irradiation of the tail of the $n-\pi^{*}$ absorbance of $Z-4$ pzMe switched it back to $>98 \% E$ isomer. Thus, 4pzMe could be quantitatively switched in both directions, a result which surpassed all of the state-of-the-art azo switches. ${ }^{11}$ While 4pzMe additionally exhibited an impressive thermal half-life for the $Z$ isomer (10 days), compound $4 \mathrm{pzH}$ was found to be the best performing photoswitch of the initial study in terms of $Z$ isomer half-life ( $\sim 1000$ days, vide supra). Conversely, less efficient ( $Z$ to $E$ ) switching was possible for $4 \mathbf{p z H}$, due to an overlap of the $Z$ and $E$ isomer $n-\pi^{*}$ absorbances. Given the increased understanding developed above for the features of the azoheteroaryl switches that impact thermal half-life, we sought to develop comparable understanding of changes in isomer specific transitions, and thus obtain a clear design rationale for how to obtain heteroazoaryl switches with both excellent two-way photoswitching and $Z$ isomer thermal half-life.

Frontier Molecular Orbital Analysis. First, we analyzed the energy of the frontier molecular orbitals responsible of the lowest-lying singlet excited states for the $Z$ and $E$ isomers of the heteroazoaryl photoswitches. Given that two different conformations are possible for each isomer, the frontier molecular orbitals (FMO) of each isomer were calculated at the PBE0$\mathrm{D} 3 / 6-31 \mathrm{G}^{* *}$ level of theory for both conformers, in all cases. Figure 8 shows representative HOMO $(\mathrm{H}), \mathrm{HOMO}-1(\mathrm{H}-1)$ and LUMO (L) frontier MOs for $2 \mathrm{pyH}$. Both $E$ isomer conformations are planar, and differ only in the rotational orientation of the heteroaromatic ring, relative to the azophenyl group. These two conformations have very similar HOMO and 


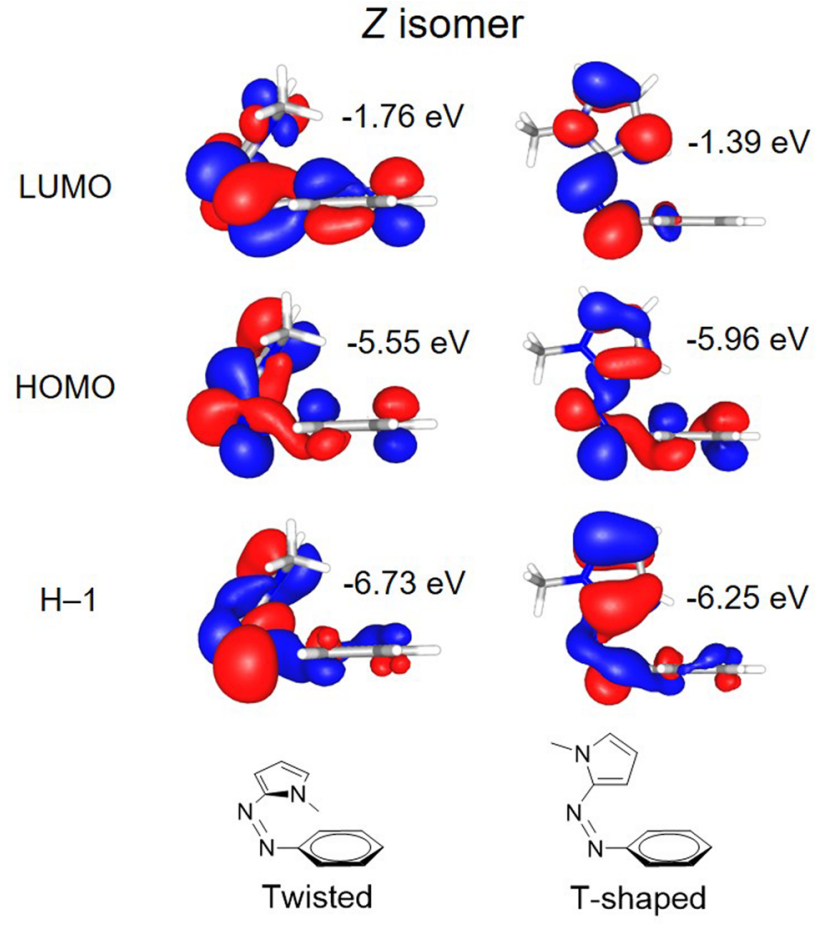

$E$ isomer

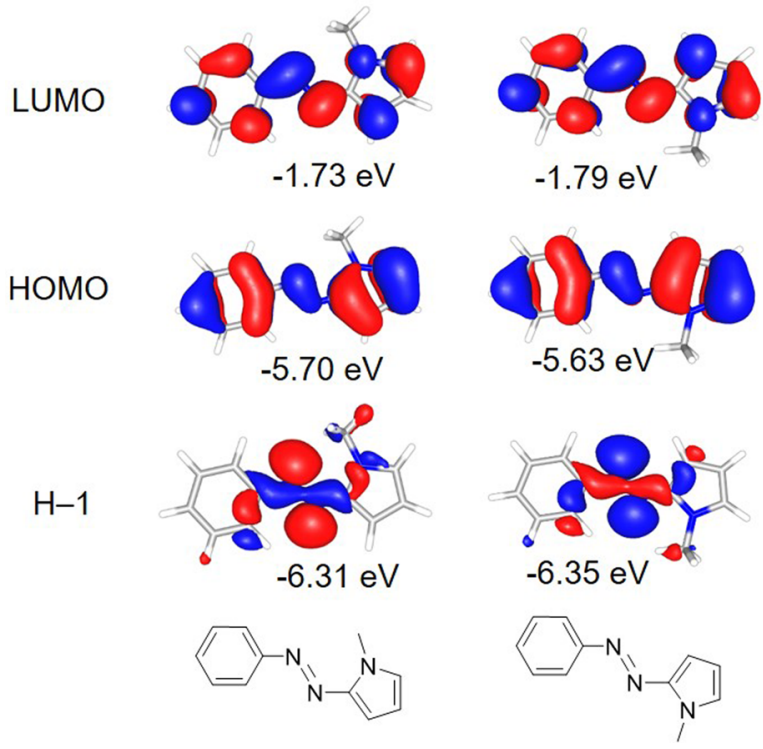

Figure 8. Frontier molecular orbitals of the representative photoswitch 2pyH in the minimum-energy orientations for $Z$ (top) and $E$ (bottom) isomers.

LUMO levels and topologies (see Figure 8), indicating that the relative orientation of the heteroarene, and thus the $E$ isomer conformation, does not have a dramatic effect on the frontier MOs (Figure S30). The HOMO and LUMO of the $E$ isomer are clearly of a $\pi$-nature whereas the HOMO- 1 is an $n$-type orbital. ${ }^{32}$ The conformation of the $Z$ isomer had a much more dramatic effect on the frontier MOs. While the symmetry of the HOMO and HOMO-1 mostly shows mixed $n / \pi$ character for the $Z$ isomer conformers, compounds able to form an orthogonal $\mathrm{T}$-shaped conformation (Figure 8) result in a more clearly defined $n$-type HOMO and a $\pi$-type HOMO-1 orbital due to symmetry constraints. The LUMO in all the $Z$ isomers is of a $\pi$ nature.

The significant variation in $Z$ isomer frontier MOs energy levels, as a function of conformation, can clearly be observed across the series in Figure 9, in contrast to that found for the $E$ isomer (Figure S30). For the $Z$ isomers, the photoswitches that experience the largest differences in the FMO energy between the two conformers are $2 \mathrm{pyH}, 4 \mathrm{im}, 5 \mathrm{im}, 3 \mathrm{pyH}$ and $\mathbf{5 p z H}$. These compounds contain one substituted ortho position (either $\mathrm{N}-\mathrm{Me}, \mathrm{C}-\mathrm{Me}$ or a basic $\mathrm{N}$ lone pair) and one unsubstituted position $(\mathrm{C}-\mathrm{H})$. As such, they can exhibit either a twisted or a T-shaped conformation (see Figure 8 for 2 pyH). In the $\mathrm{T}$-shaped conformation, the $n$-orbital is stabilized while the $\pi^{*}$-orbital becomes higher in energy, resulting in a considerable increase of the HOMO-LUMO energy gap.

Following this analysis, we sought to correlate the frontier molecular orbitals with their associated electronic transition energy. The 20 lowest-lying singlet excited states were computed for each conformer under the time-dependent density functional theory (TDDFT) framework ${ }^{33}$ at the PBE0/6-31G** level. The reliability of the PBE0 functional was assessed by comparison with other popular hybrid functionals (see Tables S5-S8 in the SI). ${ }^{34}$ The frontier molecular orbital gaps were compared to the associated electronic transition energies in order to analyze the amount of monodeterminantal character of the most important excited states in $Z$ and $E$ isomers (see SI). For the $E$ isomers, the azoheteroarene photoswitches follow a linear correlation between the $S_{1}$ energy and the $(\mathrm{H}-1)-\mathrm{L}$ gap (Figure S31a), suggesting that this transition is best described by the oneelectron promotion for the $\mathrm{H}-1$ to the LUMO. The $\mathrm{H}-1$ is the typical $n$-type lone pair orbital for the azo group (Figure 8 ). We note that while the ground state is usually well represented by a single Slater-determinant, the excited state often consists of several determinants. ${ }^{35}$ Thus, depending on the mixture of configurations, the size of the two electron-terms and the orbital energy differences, the $\mathrm{H} \rightarrow \mathrm{L}$ transition is not necessarily the lowest in energy. ${ }^{36}$ The $S_{2}$ transition for the $E$ isomer, in turn, corresponds to the $\mathrm{H} \rightarrow \mathrm{L}$ electronic transition, with a linear correlation between the $\mathrm{S}_{2}$ energy and the $\mathrm{H}-\mathrm{L}$ gap (Figure S31b). For the $Z$ isomers, the lowest-lying $S_{1}$ excited state correlates excellently with the $\mathrm{H}-\mathrm{L}$ gap in all compounds (Figure S32a); the correlation being indicative of the monodeterminantal character of this electronic transition. Conversely, the $S_{2}$ excited state is calculated as being multiconfigurational, showing a poor correlation with the $\mathrm{H}-1 \rightarrow \mathrm{L}$ gap (Figure S32b). This analysis shows that care should be taken in the analysis of optical transitions relative to HOMO-LUMO energies.

Experimental Photoswitching. In order to provide a larger experimental data set to compare with this computational analysis, UV-vis spectra and photoswitching experiments were recorded for each of the new photoswitches in acetonitrile. Since the best photoconversions were generally achieved with $355 \mathrm{~nm}(E-Z)$ and $532 \mathrm{~nm}(Z-E)$ irradiation, these wavelengths were principally used in the switching experiments (see Table 3). Azopyrazole E-5pzH could be quantitatively switched to its $Z$ isomer using $355 \mathrm{~nm}$ irradiation, while the majority of other compounds were switched to photostationary states (PSSs) containing a mixture of isomers. Z-3pyMe could be near-quantitatively (98\%) switched back to its $E$ isomer using $532 \mathrm{~nm}$, while the majority of other compounds gave PSSs containing a mixture of isomers, due to overlapping 


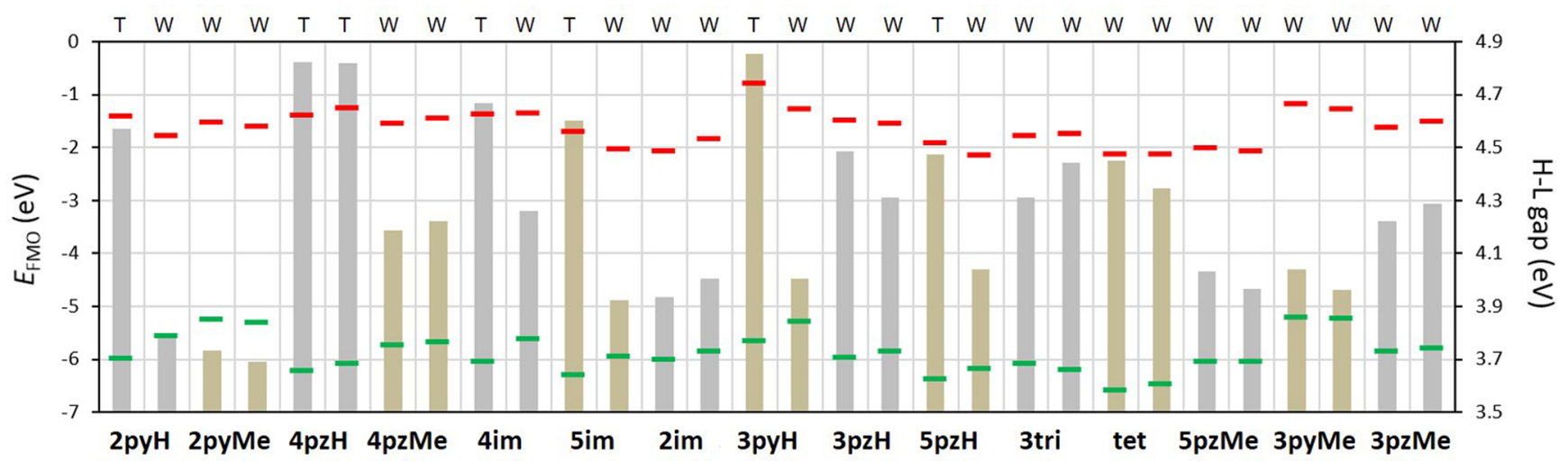

Figure 9. Frontier molecular orbital (FMO) diagram for the $Z$ isomers. FMO energies are plotted as green (HOMO) and red (LUMO) lines, with the computed energy given by the left-hand side axis. HOMO-LUMO energy gaps are displayed as bars, with the computed energy given by the right-hand side axis. Each compound is associated with two sets of data which correspond to the two possible conformers. Twisted and T-shaped arrangements are labeled as $\mathrm{W}$ and $\mathrm{T}$, respectively.

Table 3. Photostationary States and Spectral Data for the Azoheteroarenes ${ }^{a}$

\begin{tabular}{|c|c|c|c|c|c|c|c|c|c|c|}
\hline & \multicolumn{2}{|c|}{ photostationary states } & \multicolumn{2}{|c|}{$E$ isomer $\pi-\pi^{*}$} & \multicolumn{2}{|c|}{$E$ isomer $n-\pi^{*}$} & \multicolumn{2}{|c|}{$Z$ isomer $\pi-\pi^{*}$} & \multicolumn{2}{|c|}{$Z$ isomer $n-\pi^{*}$} \\
\hline & $E-Z(\% Z)$ & $Z-E(\% E)$ & $\begin{array}{c}\lambda_{\max } / \\
\mathrm{nm}\end{array}$ & $\begin{array}{l}10^{-2} \times \varepsilon / \\
\mathrm{M}^{-1} \mathrm{~cm}^{-1}\end{array}$ & $\lambda_{\max } / \mathrm{nm}$ & $\begin{array}{l}10^{-2} \times \varepsilon / \\
\mathrm{M}^{-1} \mathrm{~cm}^{-1}\end{array}$ & $\begin{array}{c}\lambda_{\max } / \\
\mathrm{nm}\end{array}$ & $\begin{array}{l}10^{-2} \times \varepsilon / \\
\mathrm{M}^{-1} \mathrm{~cm}^{-1}\end{array}$ & $\begin{array}{c}\lambda_{\max } / \\
\mathrm{nm}\end{array}$ & $\begin{array}{l}10^{-2} \times \varepsilon / \\
\mathrm{M}^{-1} \mathrm{~cm}^{-1}\end{array}$ \\
\hline tet & $76 \pm 2$ & $72 \pm 3$ & 310 & $167 \pm 15$ & 442 & $3.73 \pm 0.34$ & $\sim 290$ & $24.3 \pm 2.2^{c}$ & 428 & $10.1 \pm 0.92^{c}$ \\
\hline 3 tri & $90 \pm 2$ & $84 \pm 3$ & 310 & $187 \pm 22$ & 438 & $5.05 \pm 0.59$ & 272 & $48.9 \pm 5.7^{c}$ & 434 & $12.7 \pm 1.5^{c}$ \\
\hline $3 \mathrm{pzH}$ & $>98$ & $97 \pm 3$ & 320 & $146 \pm 17$ & 425 & $7.78 \pm 0.91$ & 272 & $90.8 \pm 11$ & 422 & $10.8 \pm 1.3$ \\
\hline $5 \mathrm{pzH}$ & $>98$ & $79 \pm 3$ & 341 & $162 \pm 20$ & 425 & $6.76 \pm 0.81$ & 289 & $69.4 \pm 8.4$ & 430 & $8.73 \pm 1.1$ \\
\hline 3pzMe & $96 \pm 2$ & $87 \pm 3$ & 325 & $174 \pm 20$ & 428 & $6.12 \pm 0.72$ & 286 & $51.5 \pm 6.0^{c}$ & 438 & $13.6 \pm 1.6^{c}$ \\
\hline $5 p z M e$ & $94 \pm 2$ & $86 \pm 3$ & 340 & $186 \pm 21$ & 435 & $8.54 \pm 0.95$ & 296 & $55.3 \pm 6.2^{c}$ & 451 & $22.1 \pm 2.5^{c}$ \\
\hline 3руH & $77 \pm 3$ & $85 \pm 5$ & 363 & $193 \pm 18$ & $\sim 410^{b}$ & $b$ & 311 & $87.5 \pm 7.9^{d}$ & 405 & $14.2 \pm 1.3^{d}$ \\
\hline 3pyMe & $85 \pm 3$ & $98 \pm 2$ & 345 & $166 \pm 15$ & $\sim 410^{b}$ & $b$ & 300 & $52.0 \pm 4.6^{d}$ & 462 & $33.2 \pm 2.9^{d}$ \\
\hline 2 pyMe $^{e}$ & $85 \pm 3^{h}$ & $>98$ & 394 & $282 \pm 18$ & $\sim 430^{b}$ & $b$ & 346 & $70.4 \pm 5.7^{d}$ & 479 & $60.8 \pm 4.5^{d}$ \\
\hline $2 \mathrm{pyH}^{e}$ & $84 \pm 2^{h}$ & $82 \pm 3$ & 385 & $221 \pm 18$ & $\sim 413^{b}$ & $b$ & 333 & $162 \pm 13^{c}$ & 423 & $15.7 \pm 1.3^{c}$ \\
\hline $4 \mathrm{pzMe}^{e}$ & $>98$ & $>98$ & 335 & $227 \pm 18$ & 425 & $9.63 \pm 0.76$ & 296 & $57.7 \pm 4.6$ & 441 & $23.0 \pm 1.8$ \\
\hline $4 \mathrm{pzH}^{e}$ & $>98$ & $70 \pm 3$ & 328 & $166 \pm 13$ & 417 & $6.40 \pm 0.51$ & 275 & $80.6 \pm 6.4$ & 403 & $6.66 \pm 0.53$ \\
\hline $2 \mathrm{im}^{f}$ & $>98^{i}$ & - & 363 & 170 & $\sim 450$ & 12.6 & - & - & - & - \\
\hline $4 \mathrm{im}^{g}$ & $>95^{j}$ & - & 336 & 148 & - & - & - & - & - & - \\
\hline $\operatorname{Sim}^{g}$ & $98^{j}$ & $45^{k}$ & 362 & 258 & - & - & - & - & - & - \\
\hline
\end{tabular}

${ }^{a_{T}}$ The position of the band $\left(\lambda_{\max }\right.$ in $\left.\mathrm{nm}\right)$ and the corresponding extinction coefficient $\left(\varepsilon\right.$ in $\left.\mathrm{M}^{-1} \mathrm{~cm}^{-1}\right)$ are indicated. See SI for UV-vis spectra. $b_{n-\pi^{*}}$ absorbance appears as a shoulder on the $\pi-\pi^{*}$ absorbance, hence $\varepsilon$ cannot be obtained. ${ }^{c}$ These values are for the extracted pure $Z$ isomer.

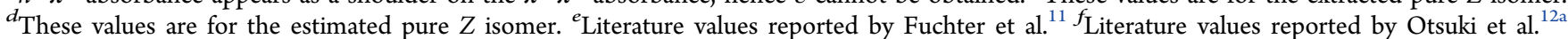

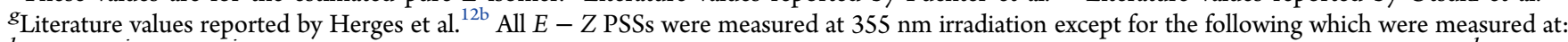
${ }^{h} 415 \mathrm{~nm} .{ }^{i} 363 \mathrm{~nm} .{ }^{j} 365 \mathrm{~nm}$. All $Z-E$ PSS were measured at $532 \mathrm{~nm}$ irradiation except for the following which was measured at: ${ }^{k} 455 \mathrm{~nm}$ irradiation.

absorbances between $E$ and $Z$ isomers at this wavelength. Notably, however, one of the azopyrazoles newly synthesized for this study-E-3pzH-could be quantitatively switched to its $Z$ isomer $(>98 \%)$ with $355 \mathrm{~nm}$ irradiation and nearquantitatively (97\%) switched back to the $E$ isomer with 532 $\mathrm{nm}$ irradiation. Theoretical calculations for Z-3pzH predict conformers slightly distorted from a perfectly $\mathrm{T}$-shaped disposition (Figure S33), resulting in an increased oscillator strength for the $n-\pi^{*}$ transition compared to, e.g., the analogous $4 \mathbf{p z H}$ system (Table 3 ), thus allowing improved switching at $532 \mathrm{~m}$ for $Z-3 \mathbf{p z H}$. This, combined with its long half-life for thermal isomerization $\left(t_{1 / 2}=74 \mathrm{~d}\right.$ at $\left.25{ }^{\circ} \mathrm{C}\right)$, makes it an extremely desirable photoswitch (i.e., comparable photoconversions to $4 \mathrm{pzMe},{ }^{11}$ but with increased $Z$ isomer thermal stability). PSS compositions and extracted $Z$ isomer spectra (Figures S11 and S12), were determined as described in the SI (and as demonstrated in Figures S4 to S10). From this, absorption maxima $\left(\lambda_{\max }\right)$ and molar extinction coefficients $(\varepsilon)$ were obtained for the $\pi-\pi^{*}$ and $n-\pi^{*}$ absorbances of both isomers of each compound (Table 3 ).

$n-\pi *$ Electronic Transition. TDDFT calculations were used to further rationalize the experimental results obtained, and in light of the analysis above. Each conformer was treated independently, and the effect of the conformation (T-shaped, twisted, etc.) was analyzed throughout. In the planar azoheteroarene $E$ isomers, a mirror plane exists through the plane generated by the azo function (for both conformers), and therefore the $n-\pi^{*}$ transition becomes symmetry-forbidden at the equilibrium geometry. Indeed, negligible intensity is computed for the $n-\pi^{*}(\mathrm{H}-1 \rightarrow \mathrm{L})$ transition of the $E$ isomer (Table S5). Persico and co-workers ${ }^{37}$ reported that the intensity of the $n-\pi^{*}$ absorption band for E-azobenzene depends on the effect that vibrational motions have on the electronic structure. Therefore, the anharmonicity, the thermal distributions, and the solvent effects all have to be taken into account. Vibrational and rotational motion leads to a 
population of molecules away from the equilibrium geometry, which provide weakly allowed $n-\pi^{*}$ transitions and correspondingly a small absorbance. This rationale is clearly also relevant for azoheteroarenes, with a weak $n-\pi^{*}$ transition observed experimentally for the $E$ isomers of all the compounds studied (Table 3).

The $Z$ isomer $n-\pi^{*}(\mathrm{H} \rightarrow \mathrm{L})$ transition, on the other hand, is computed from weak to moderately intense, depending on the photoswitch and conformation under analysis (Table S6). The most important feature affecting the magnitude of the $n-\pi^{*}$ band intensity in the $Z$ isomer is the potential to form a $\mathrm{T}$-shaped conformation. In the perfect T-shaped conformer, as in the planar $E$ isomer, a mirror plane exists through the plane of the azo function and, therefore, the transition becomes symmetry-forbidden at the equilibrium geometry. As a result, all the compounds with the azoheteroaryl group orthogonal to the benzene ring (CCNN dihedral $\approx 90^{\circ}$, T-shaped) are computed to have a singlet $S_{1}$ excitation with negligible oscillator strength (Figure 10). Conversely, the photoswitches

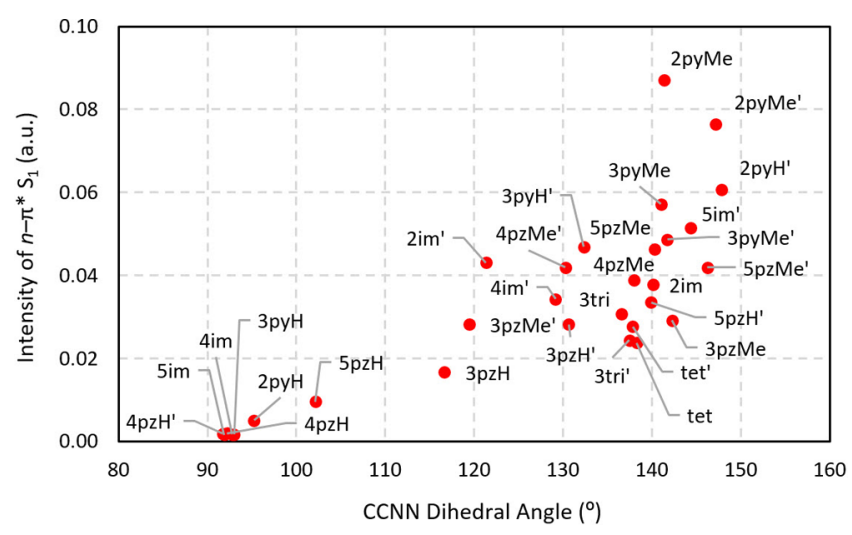

Figure 10. Correlation between the intensity of the lowest-lying $S_{1}$ excited state of $n-\pi^{*}$ nature with respect to the dihedral between the benzene and the azo group in the $Z$ isomer. The two possible conformers for each isomer are included.

that cannot adopt a $\mathrm{T}$-shaped conformation exhibit an $\mathrm{S}_{1}$ state with oscillator strengths up to $f=0.090$. The correlation of the CCNN dihedral angle to the computed intensity of the $n-\pi^{*}$ transition, for all the photoswitches studied, can be seen in Figure 10. Such conformation-induced tuning of the magnitude of the $n-\pi^{*}$ transition intensity is clearly observable from the experimental data. For example, the $Z$ isomers of photoswitches with two ortho-methyls on the heteroarene (5pzMe, 3pyMe) cannot adopt a T-shaped conformation (vide supra), and therefore had a considerably larger $n-\pi^{*}$ absorbance than their non- or singly $o$-methylated analogues (Table 3 ). Likewise, the presence of two heteroaryl basic nitrogens adjacent to the azo group (tet, 3tri), or one basic nitrogen and one ortho-methyl (3pzMe), increased the intensity of the $Z$ isomer $n-\pi^{*}$ band relative to that of the $E$ isomer (Table 3 ), due to an increasingly twisted conformation. Since the T-shaped conformation is additionally responsible for an increased $\mathrm{H}-\mathrm{L}$ band gap (vide supra), compounds exhibiting such a conformation in their $Z$ isomer should result in a shift of the $n-\pi^{*}$ band to higher energies. Experimentally this is indeed the case-for example, the $\lambda_{\max }$ for the $n-\pi^{*}$ transitions of $Z-3 \mathbf{p y H}$ and $Z-5 \mathrm{pzH}$ occur at shorter wavelengths than those of $Z$-3pyMe and Z-5pzMe, respectively.
An increase in the number of $\mathrm{N}$ atoms is predicted to result in a systematic stabilization of the HOMO and LUMO for the $Z$ isomers, and a global decrease in the HOMO-LUMO gap (Figure 9). When comparing the $Z$-isomers of $\mathbf{3 p z H}, \mathbf{4} \mathbf{p z H}$, 4im, 3tri and tet, there is a general decrease in energy of the $n-\pi^{*}$ electronic transition upon increasing the number of $\mathrm{N}$ atoms as calculated by TDDFT (Table S6). For example, the $n-\pi^{*}$ electronic excitation is predicted to change from $409 \mathrm{~nm}$ in $4 \mathrm{pzH}$ to $455 \mathrm{~nm}$ in 3 tri, although little difference is found moving to tet $(453 \mathrm{~nm})$. This trend is supported by the experimental data: $\lambda_{\max }=403,434$, and $428 \mathrm{~nm}$ for $4 \mathrm{pzH}$, 3tri and tet, respectively. In terms of conjugation effects resulting from heteroaryl connectivity, a consistent shift of the $n-\pi^{*}(\mathrm{H}$ $\rightarrow \mathrm{L}$ ) transition to longer wavelengths was predicted for compounds going from "partial" to "complete" heteroarene conjugation (Table S6 and Figure 6). For instance, the $n-\pi^{*}$ excitation of "partial"-conjugated 3-azopyrroles $3 \mathrm{pyH}$ and 3pyMe is calculated at 438 and $473 \mathrm{~nm}$, respectively, which moves to 458 and $490 \mathrm{~nm}$ for the "complete"-conjugated 2azopyrroles 2pyH and 2pyMe. This also holds for the "partial"conjugated 3- (3pzH and 3pzMe) and 4-azopyrazoles (4pzH and 4pzMe) compared to the "complete"-conjugated 5pyrazole compounds ( $5 \mathrm{pzH}$ and $\mathbf{5 p z M e}$ ). These trends are in excellent agreement with the experimental position of the $n-\pi^{*}$ band in the $Z$ isomers gathered in Table 3 .

$\pi-\pi^{*}$ Electronic Transition. For the majority of the compounds studied, the $\pi-\pi^{*}$ electronic transition of the $E$ isomer was computed to exhibit a moderately intense absorbance band with oscillator strength $f=0.5-0.9$. Since the $\pi-\pi^{*}$ electronic transition of the $E$ isomers is assigned to a HOMO to LUMO transition $(\mathrm{H} \rightarrow \mathrm{L})$, the computed $\mathrm{H}-\mathrm{L}$ band gap trends can be compared to the $\lambda_{\max }$ position of the $E$ isomer $\pi-\pi^{*}$ transition. When comparing compounds where the $\mathrm{N}-\mathrm{Me}$ group is located in the same position relative to the azo linkage ( $3 \mathrm{pzH}$ vs 3 tri vs tet), a systematic increase in the energy of the $\pi-\pi^{*}$ transition is computed as the number of nitrogen atoms increases: from $3.99 \mathrm{eV}(311 \mathrm{~nm})$ in $3 \mathbf{p z H}$ to $4.09 \mathrm{eV}(303 \mathrm{~nm})$ in tet. When considering compounds with two nitrogen atoms in the heterocyclic ring, but positioned differently (4pzH vs $4 \mathrm{im}$ vs $3 \mathrm{pzH})$, the $\pi-\pi^{*}$ bands of $4 \mathrm{pzH}$ and $4 \mathrm{im}$ were predicted to have comparable $\pi-\pi^{*}$ transition energies of 315 and $319 \mathrm{~nm}$ (3.94 and $3.89 \mathrm{eV}$ ), respectively, both lower than $3 \mathbf{p z H}$, which has a calculated transition energy of $311 \mathrm{~nm}(3.99 \mathrm{eV})$. These trends are supported by the experimental data, although, as for the $n-\pi^{*}$ band, little/no difference is observed for 3tri versus tet: $\lambda_{\max }=336,328,320$, 310 , and $310 \mathrm{~nm}$ for E-4im, 4pzH, 3pzH, 3tri and tet respectively. The $\pi-\pi^{*}$ band of 3 pyMe and $3 \mathrm{pyH}$ was less redshifted than for the 2-azopyrrole analogues (2pyMe and $2 \mathrm{pyH}$ ); a result of "partial" versus "complete" conjugation of the azo group with the heteroarene (see Figure 6). For similar reasons, the 5-azopyrazole analogue $5 \mathrm{pzH}$ had red-shifted $\pi-\pi^{*}$ absorbances relative to the 3-azopyrazole and 4azopyrazole analogues ( $3 \mathrm{pzH}$ and $4 \mathrm{pzH})$, while a small redshift was seen for bis(o-methylated) 5-azopyrazole E-5pzMe relative to 4-azopyrazole E-4pzMe. The calculated data are in excellent agreement with the experimental trends for the 3-, 4and 5-azopyrazoles, whereas the significant conformationaldependence of the $\pi-\pi^{*} S_{2}$ band position (Table S5) hinders a full comparison between 3- and 2-azopyrroles.

Band Separation. Given that the extent of photoswitching in the PSS is primarily a function of the overlap of bands in the absorption spectrum, and the corresponding intensity of the 
Table 4. Experimental and Theoretical Separation (Values in nm Are Given as $Z$ Minus $E$ ) of the $n-\pi^{*}$ and $\pi-\pi^{*}$ Bands for the $Z$ and $E$ Isomers

\begin{tabular}{|c|c|c|c|c|c|c|}
\hline \multirow[b]{2}{*}{ compound } & \multicolumn{2}{|c|}{$n-\pi^{*}$ separation $(Z-E)$} & \multicolumn{2}{|c|}{$\pi-\pi^{*}$ separation $(Z-E)$} & \multicolumn{2}{|c|}{$n-\pi^{*}(Z)-\pi-\pi^{*}(E)$} \\
\hline & experimental & theoretical $^{a}$ & experimental & theoretical $^{a}$ & experimental & theoretical $^{a}$ \\
\hline $2 \mathrm{pyH}$ & 10 & 16 & -52 & -38 & 38 & 113 \\
\hline 2pyMe & 49 & 50 & -48 & -29 & 85 & 135 \\
\hline $4 \mathrm{pzH}$ & -14 & -38 & -53 & -36 & 75 & 94 \\
\hline $4 \mathrm{pzMe}$ & 16 & 15 & -39 & -30 & 106 & 141 \\
\hline $4 \mathrm{im}$ & - & -15 & - & -28 & - & 127 \\
\hline $5 \mathrm{im}$ & - & 8 & - & -29 & - & 119 \\
\hline $2 \mathrm{im}$ & - & 22 & - & -11 & - & 145 \\
\hline 3руH & -5 & -9 & -52 & -32 & 42 & 90 \\
\hline $3 \mathrm{pzH}$ & -3 & -13 & -48 & -19 & 102 & 143 \\
\hline $5 \mathrm{pzH}$ & 5 & 6 & -52 & -23 & 89 & 135 \\
\hline 3 tri & -4 & -25 & -38 & -23 & 124 & 135 \\
\hline tet & -14 & -28 & -20 & -16 & 118 & 152 \\
\hline $5 p z M e$ & 16 & 28 & -44 & -12 & 111 & 150 \\
\hline 3pyMe & 51 & 22 & -46 & -9 & 115 & 148 \\
\hline $3 \mathrm{pzMe}$ & 10 & -2 & -39 & -6 & 113 & 117 \\
\hline
\end{tabular}

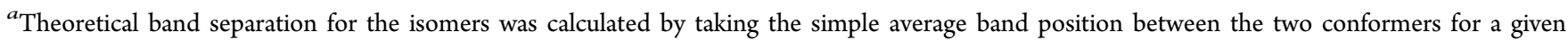
isomer.

relevant transitions, it is important to fully understand the structure-property relationships that underpin band separation. While the discussion above individually compares the position and intensity of the main $n-\pi^{*}$ and $\pi-\pi^{*}$ transitions as a function of both the azoheteroaryl photoswitch and isomer dependent conformations, a comparison of the computed versus experimental band separation is warranted. Such a comparison is shown in Table 4. In general, the computed band separations qualitatively correlate with those observed experimentally. By comparing compounds with and without orthomethyl substitution, a significant enhancement especially of the $Z-E$ photoconversion is achieved when substituting the ortho $\mathrm{H}$ by a methyl group. For instance, $\%(Z-E)$ photoconversion increases from $82,85,70$ and $79 \%$ in $2 \mathbf{p y H}, 3 \mathbf{p y H}, 4 \mathbf{p z H}$ and 5 pzH, respectively, to $>98,98,>98$ and $86 \%$ for 2 pyMe, 3pyMe, 4pzMe and 5pzMe (Table 3). This improvement in the photoconversion efficiency is a result of $n-\pi^{*}$ band separation between $Z$ and $E$ isomers, which increases upon moving from $\mathrm{H}$-substituted to methyl-substituted photoswitches (Table 4), a result of the effect of the increasingly twisted conformation of the $Z$ isomer (vide supra). In the case of $4 \mathrm{pzH} / 4 \mathrm{pzMe}$, inversion of the $n-\pi^{*}$ band positions between $E$ and $Z$ is found both experimentally and theoretically. Pyrrole heteroaromatic switches show in general poorer $E-Z$ photoconversion efficiencies with respect to the pyrazole analogues, a result of the difference between the $n-\pi^{*}$ band of the $Z$ isomer and the $\pi-\pi^{*}$ of $E$ isomer (Table 4). For example, the experimental $n-\pi^{*}(Z)$ minus $\pi-\pi^{*}(E)$ difference is recorded to increase from $42 \mathrm{~nm}$ in $3 \mathrm{pyH}$ to $102 \mathrm{~nm}$ in 3 pzH, thus explaining the $E-Z$ photoconversion improvement from 77 to $>98 \%$.

While intrinsically good band separation enables improved photoswitching, judicious choice of the irradiation wavelength, particularly to spectral regions significantly outside of the absorption maxima, can allow for further improvement in the completeness of photoswitching (albeit with a decrease in quantum yield). A key case in point in this study is photoswitch $3 \mathrm{pzH}$. Using light at $532 \mathrm{~nm}$ (the $n-\pi^{*} \lambda_{\max }$ of $Z-3 \mathrm{pzH}$ is 422 $\mathrm{nm}$ ), excellent $Z-E$ photoisomerisation was achieved for 3 pzH $(97 \% E)$, although a relatively long irradiation time $(\sim 15$ min) was required to reach the PSS. We tentatively assign this to a difference in oscillator strengths between $E$ and $Z$ isomers for $3 \mathrm{pzH}$ at $532 \mathrm{~nm}$ : the $Z-3 \mathbf{p z H}$ conformation (which is slightly distorted from a perfectly $\mathrm{T}$-shaped disposition; Figure S33) results in an increased oscillator strength for the $n-\pi^{*}$ transition at $532 \mathrm{~nm}$ compared to the planar E-3pzH. Thus, once again, the chemical structure and conformation of the potential photoswitch can help us comprehend and design compounds that can achieve better photoconversions.

\section{CONCLUSIONS}

We present a systematic computational and experimental study on the azoheteroarene photoswitches: photochromic azoaryl molecules consisting of one five-membered heteroaromatic ring and one benzene ring. Modification of the type of the heteroaromatic ring, as well as its positioning (relative to the azo group) and substitution has been found to have a huge effect on the performance of this class of switch. This is most notable in the $Z$ isomer thermal half-life data, where we identify compounds with half-lives ranging from seconds to hours, to days and to years. In-depth computational study has revealed the thermal $Z-E$ isomerization rate to be determined by both thermodynamic $(Z-E$ isomer relative stability) and kinetic (activation barrier) factors, which are, in turn, influenced by the structure and conformation of the photoswitch in question. Conformation perhaps plays the largest role, where the compounds with the longest isomerization half-lives adopt a T-shaped ground state $Z$ isomer conformation and proceed through a T-shaped isomerization pathway. Noncovalent interaction analysis can readily explain the stabilization or destabilization of such conformations.

Conversely, the most complete photoswitching is achieved for compounds that have a twisted (rather than T-shaped) $Z$ isomer conformation; where such a conformation is achieved through the use of substituents or basic nitrogen atoms in the ortho positions. Such twisting particularly results in an increase in oscillator strength for the $n-\pi^{*}$ band of the $Z$ isomer and therefore allows for the selection of wavelengths that can selectively address each isomer. Ultimately, to achieve complete bidirectional photoswitching and a long $Z$ isomer half-life, a 
balance must be found between these two conformational states. In this study, such a balance is exemplified by the newly discovered azopyazole $3 \mathbf{p z H}$, which can be quantitatively switched to its $Z$ isomer ( $>98 \%$ ) with $355 \mathrm{~nm}$ irradiation, near-quantitatively (97\%) switched back to the $E$ isomer with $532 \mathrm{~nm}$ irradiation, and has a very long half-life for thermal isomerization $\left(t_{1 / 2}=74 \mathrm{~d}\right.$ at $\left.25^{\circ} \mathrm{C}\right) . Z-3 \mathbf{p z H}$ has a conformation only slightly distorted from a perfectly $\mathrm{T}$-shaped disposition (Figure S33), which retains the slow thermal isomerization of the $Z$ isomer, but results in an increased oscillator strength for the $n-\pi^{*}$ transition compared to, e.g., the analogous 4 pzH system. ${ }^{11}$

We also show the Wiberg index to be suitably useful as a predictive measure for estimating thermal half-lives, allowing for large numbers of compounds to be screened without the need to obtain free energies for the $E$ and $Z$ isomer conformations and TSs, which have high computational cost. Although outliers are present in our analysis, which limit quantitative comparison, our data (see Figure 7) clearly shows that assessment of the Wiberg index gives a useful "first approximation" as to whether a given switch is likely to have a short, medium or long half-life. As such, this should allow identification of photoswitches that would be suited to particular applications, where either short or long thermal half-lives may be required.

Given the large tunability of their properties, the predictive nature of their performance, and the other functional opportunities $^{15,17 \mathrm{a}}$ afforded by usage of a heteroaromatic system, we believe the azoheteroaryl photoswitches to have huge potential in a wide range of optically addressable applications. The approaches hereby presented provide a means to rationally and rapidly design novel photoswitches to achieve a given performance requirement.

\section{ASSOCIATED CONTENT}

\section{S Supporting Information}

The Supporting Information is available free of charge on the ACS Publications website at DOI: 10.1021/jacs.6b11626.

Synthetic methods, NMR and crystallographic data, photochemical methods including additional spectra and kinetics, and computational details (PDF) Crystal data (CIF)

\section{AUTHOR INFORMATION}

\section{Corresponding Authors}

*contrera@lct.jussieu.fr

*m.fuchter@imperial.ac.uk

\section{ORCID ${ }^{\circ}$}

Matthew J. Fuchter: 0000-0002-1767-7072

\section{Author Contributions}

\#C and CEW contributed equally to this work.

\section{Notes}

The authors declare no competing financial interest.

Raw data files are openly available at DOI: 10.6084/ m9.figshare.4287302.

\section{ACKNOWLEDGMENTS}

MJF and CEW thank the Engineering and Physical Sciences Research Council for funding. JC acknowledges the Spanish Ministry of Education, Culture, and Sport (MECD) for an FPU grant. We also thank Dr Kuimova (Imperial College London) for equipment access.

\section{REFERENCES}

(1) (a) Gostl, R.; Senf, A.; Hecht, S. Chem. Soc. Rev. 2014, 43, 19821996. (b) Bléger, D.; Hecht, S. Angew. Chem., Int. Ed. 2015, 54, 11338-11349. (c) Szymański, W.; Beierle, J. M.; Kistemaker, H. A. V.; Velema, W. A.; Feringa, B. L. Chem. Rev. 2013, 113, 6114-6178. (d) Feringa, B. L.; Browne, W. R. Molecular Switches; VCH: Weinheim, 2011. (e) Russew, M.-M.; Hecht, S. Adv. Mater. 2010, 22, 3348-3360. (f) Kucharski, T. J.; Ferralis, N.; Kolpak, A. M.; Zheng, J. O.; Nocera, D. G.; Grossman, J. C. Nat. Chem. 2014, 6, 441-447. (g) Zhitomirsky, D.; Cho, E.; Grossman, J. C. Adv. Energy Mater. 2016, 6, 1502006. (h) Feng, Y.; Liu, H.; Luo, W.; Liu, E.; Zhao, N.; Yoshino, K.; Feng, W. Sci. Rep. 2013, 3, 3260. (i) Saydjari, A. K.; Weis, P.; Wu, S. Adv. Energy Mater. 2016, 1601622.

(2) (a) Bandara, H. M. D.; Burdette, S. C. Chem. Soc. Rev. 2012, 41, 1809-1825. (b) Beharry, A. A.; Woolley, G. A. Chem. Soc. Rev. 2011, 40, 4422-4437.

(3) (a) Bastianelli, C.; Caia, V.; Cum, G.; Gallo, R.; Mancini, V. J. Chem. Soc., Perkin Trans. 2 1991, 679-683. (b) Irie, M.; Fukaminato, T.; Matsuda, K.; Kobatake, S. Chem. Rev. 2014, 114, 12174-12277. (c) Feng, W.; Luo, W.; Feng, Y. Nanoscale 2012, 4, 6118-6134. (d) Luo, W.; Feng, Y.; Cao, C.; Li, M.; Liu, E.; Li, S.; Qin, C.; Hu, W.; Feng, W. J. Mater. Chem. A 2015, 3, 11787-11795.

(4) (a) Beharry, A. A.; Sadovski, O.; Woolley, G. A. J. Am. Chem. Soc. 2011, 133, 19684-19687. (b) Bléger, D.; Schwarz, J.; Brouwer, A. M.; Hecht, S. J. Am. Chem. Soc. 2012, 134, 20597-20600. (c) Yang, Y.; Hughes, R. P.; Aprahamian, I. J. Am. Chem. Soc. 2012, 134, 1522115224. (d) Siewertsen, R.; Neumann, H.; Buchheim-Stehn, B.; Herges, R.; Näther, C.; Renth, F.; Temps, F. J. Am. Chem. Soc. 2009, 131, 15594-15595.

(5) (a) Velema, W. A.; Szymanski, W.; Feringa, B. L. J. Am. Chem. Soc. 2014, 136, 2178-2191. (b) Broichhagen, J.; Frank, J. A.; Trauner, D. Acc. Chem. Res. 2015, 48, 1947-1960.

(6) (a) Samanta, S.; Beharry, A. A.; Sadovski, O.; McCormick, T. M.; Babalhavaeji, A.; Tropepe, V.; Woolley, G. A. J. Am. Chem. Soc. 2013, 135, 9777-9784. (b) Samanta, S.; McCormick, T. M.; Schmidt, S. K.; Seferos, D. S.; Woolley, G. A. Chem. Commun. 2013, 49, 1031410316.

(7) Knie, C.; Utecht, M.; Zhao, F.; Kulla, H.; Kovalenko, S.; Brouwer, A. M.; Saalfrank, P.; Hecht, S.; Bléger, D. Chem. - Eur. J. 2014, 20, $16492-16501$.

(8) Other successful attempts to red shift azo photoswitch absorbance include through $\mathrm{BF}_{2}$-coordination. See, e.g., refs $4 \mathrm{c}$ and Yang, Y.; Hughes, R. P.; Aprahamian, I. J. Am. Chem. Soc. 2014, 136, 13190-13193.

(9) van Dijken, D. J.; Kovaříček, P.; Ihrig, S. P.; Hecht, S. J. Am. Chem. Soc. 2015, 137, 14982-14991.

(10) Hammerich, M.; Schütt, C.; Stähler, C.; Lentes, P.; Röhricht, F.; Höppner, R.; Herges, R. J. Am. Chem. Soc. 2016, 138, 13111-13114.

(11) Weston, C. E.; Richardson, R. D.; Haycock, P. R.; White, A. J. P.; Fuchter, M. J. J. Am. Chem. Soc. 2014, 136, 11878-11881.

(12) (a) Otsuki, J.; Suwa, K.; Narutaki, K.; Sinha, C.; Yoshikawa, I.; Araki, K. J. Phys. Chem. A 2005, 109, 8064-8069. (b) Wendler, T.; Schütt, C.; Näther, C.; Herges, R. J. Org. Chem. 2012, 77, 3284-3287. (c) Garcia-Amorós, J.; Díaz-Lobo, M.; Nonell, S.; Velasco, D. Angew. Chem., Int. Ed. 2012, 51, 12820-12823.

(13) Substitution at one or both of the positions of the 5-member heteroarene adjacent to the azo group are referred to as "ortho" throughout. Although this is not formally the correct usage of the term, it allows for simple comparison with the ortho-substituted azobenzenes.

(14) Stricker, L.; Fritz, E.-C.; Peterlechner, M.; Doltsinis, N. L.; Ravoo, B. J. J. Am. Chem. Soc. 2016, 138, 4547-4554.

(15) Weston, C. E.; Kraemer, A.; Colin, F.; Yildiz, Ö.; Baud, M. G. J.; Meyer-Almes, F.-J.; Fuchter, M. J. ACS Infect. Dis. 2016, DOI: 10.1021 /acsinfecdis.6b00148.

(16) Value reported in this work. 
(17) (a) Weston, C. E.; Richardson, R. D.; Fuchter, M. J. Chem. Commun. 2016, 52, 4521-4524. (b) Schütt, C.; Heitmann, G.; Wendler, T.; Krahwinkel, B.; Herges, R. J. Org. Chem. 2016, 81, 12061215.

(18) (a) Schweighauser, L.; Strauss, M. A.; Bellotto, S.; Wegner, H. A. Angew. Chem., Int. Ed. 2015, 54, 13436-13439. (b) Wang, Y.-P.; Zhang, Z.-X.; Xie, M.; Bai, F.-Q.; Wang, P.-X.; Zhang, H.-X. Dyes Pigm. 2016, 129, 100-108. (c) Ikegami, T.; Kurita, N.; Sekino, H.; Ishikawa, Y. J. Phys. Chem. A 2003, 107, 4555-4562. (d) Wazzan, N. A.; Richardson, P. R; Jones, A. C. Photochem. Photobiol. Sci. 2010, 9, 968974. (e) Dokić, J.; Gothe, M.; Wirth, J.; Peters, M. V.; Schwarz, J.; Hecht, S.; Saalfrank, P. J. Phys. Chem. A 2009, 113, 6763-6773. (f) Cembran, A.; Bernardi, F.; Garavelli, M.; Gagliardi, L.; Orlandi, G. J. Am. Chem. Soc. 2004, 126, 3234-3243.

(19) Nabati, M. J. Phys. Theor. Chem. IAU Iran 2016, 12, 325-338.

(20) Wang, Y.-T.; Liu, X.-Y.; Cui, G.; Fang, W.-H.; Thiel, W. Angew. Chem., Int. Ed. 2016, 55, 14009.

(21) Adamo, C.; Barone, V. J. Chem. Phys. 1999, 110, 6158-6170.

(22) Grimme, S.; Antony, J.; Ehrlich, S.; Krieg, H. J. Chem. Phys. 2010, 132, 154104.

(23) Francl, M. M.; Pietro, W. J.; Hehre, W. J.; Binkley, J. S.; Gordon, M. S.; Defrees, D. J.; Pople, J. A. J. Chem. Phys. 1982, 77, 3654-3665.

(24) (a) Johnson, E. R.; Keinan, S.; Mori-Sánchez, P.; ContrerasGarcía, J.; Cohen, A. J.; Yang, W. J. Am. Chem. Soc. 2010, 132, 64986506. (b) Contreras-García, J.; Johnson, E. R.; Keinan, S.; Chaudret, R.; Piquemal, J.-P.; Beratan, D. N.; Yang, W. J. Chem. Theory Comput. 2011, 7, 625-632.

(25) Assuming a 50\% population of each conformer was found to give a comparable correlation between theoretical and experimental half-life times to a Boltzmann distribution of the two possible $\mathrm{Z}$ conformers.

(26) Eyring, H. J. Chem. Phys. 1935, 3, 107-115.

(27) Only compounds with half-lives of less than 1 day at rt (2pyH, 3 pyH, 5pzMe) were studied in order to access accurate comparisons in a timely fashion. 2 pyMe was not studied due to its very short halflife.

(28) See the Supporting Information of ref 11.

(29) Hill, E. A.; Gross, M. L.; Stasiewicz, M.; Manion, M. J. Am. Chem. Soc. 1969, 91, 7381-7392.

(30) Wiberg, K. B. Tetrahedron 1968, 24, 1083-1096.

(31) Noyce, D. S.; Sandel, B. B. J. Org. Chem. 1976, 41, 3640-3642.

(32) We note that this analysis contrasts with the $n-\pi^{*}$ nature of the lowest-lying (HOMO $\rightarrow$ LUMO) electronic transition commonly assigned to $E$ isomer azo photoswitches; the $\pi-\pi^{*}$ transition being assigned to higher energies. ${ }^{2 a}$ The lowest-lying excitation is not always described by a pure HOMO $\rightarrow$ LUMO monoexcitation. We used TDDFT equations to find the best linear combination of monoexcitations and the corresponding energies. See ref 36.

(33) (a) Jamorski, C.; Casida, M. E.; Salahub, D. R. J. Chem. Phys. 1996, 104, 5134-5147. (b) Casida, M. E.; Jamorski, C.; Casida, K. C.; Salahub, D. R. J. Chem. Phys. 1998, 108, 4439-4449. (c) Petersilka, M.; Gossmann, U. J.; Gross, E. K. U. Phys. Rev. Lett. 1996, 76, 12121215.

(34) Plater, M. J.; Harrison, W. T. A.; Rzepa, H. S. J. Chem. Res. 2015, 39, 711-718.

(35) Szabo, A.; Ostlund, N. S. Modern Quantum Chemistry: Introduction to Advanced Electronic Structure Theory; Dover Publications, 1989.

(36) Casida, M. E.; Ipatov, A.; Cordova, F. In Time-Dependent Density Functional Theory; Marques, M. A. L., Ullrich, C. A., Nogueira, F., Rubio, A., Burke, K., Gross, E. U. K., Eds.; Springer: Berlin, 2006; p 243-257.

(37) Cusati, T.; Granucci, G.; Persico, M.; Spighi, G. J. Chem. Phys. 2008, 128, 194312. 